\title{
Heterogeneous Expectations and Exchange Rate Dynamics
}

\author{
Carl Chiarella ${ }^{1}$, Xue-Zhong $\mathrm{He}^{1}$ and Min Zheng ${ }^{1,2, \dagger}$ \\ ${ }^{1}$ UTS Business School, University of Technology, Sydney \\ PO Box 123, Broadway, NSW 2007, Australia \\ ${ }^{2}$ China Institute for Actuarial Science, Central University of Finance and Economics \\ 39 South College Road, Haidian District, Beijing 100081, China \\ $\dagger$ Corresponding author \\ carl.chiarella@uts.edu.au,_tony.he1@uts.edu.au,_mathzhm@gmail.com
}

\begin{abstract}
This paper presents a continuous-time model of exchange rates relying not only on macroeconomic factors but also having an investor heterogeneity component. The driving macroeconomic factor is the domestic-foreign interest rate differential, while the investor heterogeneity is described by the expectations of boundedly rational portfolio managers who use a weighted average of the expectations of fundamentalists and chartists. Within this framework, the different roles of the macroeconomic factor and investor heterogeneity on the determination of the exchange rate are examined explicitly. We show that this simple model generates very complicated market behaviour, including the existence of multiple steady state equilibria, deviations of the market exchange rate from the fundamental, and market fluctuations. Numerical simulation of the corresponding stochastic version of the model shows that the model is able to generate typical time series and volatility clustering patterns observed in exchange rate markets.
\end{abstract}

Key Words: Exchange rate; interest rate differential; heterogeneous expectations. 


\section{Introduction}

In the traditional macro-structure paradigm that underpins the influential rational expectations Dornbusch (1976) model, exchange rate determination is based on macroeconomic fundamental factors, in particular interest rates and monetary policy. However, many empirical studies of exchange rates have shown that there are often large movements in exchange rates that are apparently unexplained by macroeconomic fundamental factors. Sometimes, the market exchange rate can deviate from its fundamental equilibrium and stabilise at some non-fundamental levels for quite long periods of time. In particular, Meese and Rogoff (1983) show that a random walk model out-performs standard open economy macroeconomic models in explaining exchange rate movements. The weak explanatory power of macroeconomic factors on the exchange rate is referred to as the exchange rate puzzle or anomaly (see Lyons, 2001). Over the last two decades, an increasing number of anomalies and puzzles have been uncovered in empirical research. These anomalies or puzzles include, for example, (i) the "disconnect" puzzle, referring to the fact that exchange rates appear to be disconnected from their underlying fundamental factors most of the time (see Williamson, 1985), (ii) the "excess volatility" puzzle, whereby the volatility of exchange rates by far exceeds the volatility of their underlying economic variables (see Baxter and Stockman, 1989 and Flood and Rose, 1995), and (iii) the stylised facts referring to the non-normality, fat tails and power-law behaviour of exchange rate returns (see Lux, 1998, Lux and Marchesi, 2001 and LeBaron, 2006). We refer to De Grauwe and Grimaldi (2006) for more discussion of these empirical anomalies. The foregoing comments suggest that explanations of exchange rate movements based solely on macroeconomic fundamental factors are unsatisfactory. As a result, the attention of researchers has moved away from the examination simply of macroeconomic factors, towards taking into account the structure of the foreign exchange, in particular by considering the heterogeneity and bounded rationality of investors.

Empirical literature on survey data, in particular Ito (1990), Allen and Taylor (1990), Taylor and Allen (1992), Menkhoff (1998), Chinn and Frankel (2002) and more recently Menkhoff and Taylor (2007) provide evidence of heterogeneity and bounded rationality of the investors in exchange rate markets. These studies show the widespread influence of foreign exchange fundamental analysts who base their predictions on macroeconomic fundamental factors and technical analysts who base their predictions purely on the identification of supposedly recurring patterns in exchange rate movements. So models seeking to explain exchange rate movements should incorporate both of these elements.

Over the last two decades, the literature on models of heterogeneous agents who are boundedly rational in speculative markets has developed rapidly. In order to explain the US dollar's behaviour in the 1980s, Frankel and Froot (1986) consider an exchange rate model with three classes of agents: fundamentalists who believe the Dornbusch overshooting model, chartists who believe that the exchange rate follows a random walk, and portfolio managers who form their expectations as a weighted average of the predictions 
of the fundamentalists and chartists. The framework of Frankel and Froot departs from the rational expectations orthodoxy, by virtue of the fact that the three types of agents are boundedly rational rather than fully rational. They find that the portfolio managers learn more slowly about the model than they change it by revising a linear combination of chartist and fundamentalist views they incorporate in their own forecasts, which leads to the take-off of bubbles. In addition, the fundamental equilibrium of the model may not be stable and the exchange rate may stabilise around a value that is far from the fundamental equilibrium. Recently, some exchange rate models that study jointly the dynamics of speculative and macroeconomic forces and other exchange rate models that solely focus on the behaviour of speculators have been developed. See Westerhoff and Reitz (2003), Westerhoff (2003), Reitz and Taylor (2008) and Westerhoff (2010). These models are able to mimic the dynamics of foreign exchange markets and provide some policy implications such as central bank intervention. For the aspect of asset pricing, by incorporating bounded rationality and heterogeneity, various heterogeneous agent models have successfully explained many types of features (such as market booms and crashes, long deviations of the market price from the fundamental price), the stylized facts (such as skewness, kurtosis, volatility clustering and fat tails of returns), and various power laws (such as the long memory in return volatility) observed in financial markets. We refer the reader to Hommes (2006), Hommes and Wagener (2009), LeBaron (2006), Chiarella et al. (2009) and Westerhoff (2010) for surveys of the recent developments in this literature.

This paper seeks to revisit the Dornbusch overshooting mechanism in light of the understanding that macroeconomic factors alone are not the driver of exchange rate movements. We present a continuous-time model of exchange rate dynamics containing both macroeconomic and investor heterogeneity elements. The macroeconomic fundamental factor is determined by the domestic-foreign interest rate differential, whilst the investor heterogeneity is characterised by the expectations of the portfolio managers. From Frankel and Froot (1986, 1990a, 1990b), we borrow the idea that the expectations of the portfolio managers are a function of the weighted average of the expectations of fundamentalists and chartists. However unlike in Frankel and Froot (1986), this function is nonlinear instead of linear, the nonlinearity being due to the cautiousness of the portfolio managers as weighted expectations take extreme values. The incorporation of fundamentalists and chartists allows for the bounded rationality of the heterogeneous investors rather than the full rationality that is usually proposed and that creates an "infinite regress" problem, as argued in De Grauwe and Grimaldi (2006). Within our framework, we are able to examine explicitly the different roles of the macroeconomic fundamental factor and investor heterogeneity on the determination of the exchange rate. By the introduction of cautiousness into the expectations of the portfolio managers, we provide a channel for the market exchange rate to deviate from and fluctuate around the fundamental exchange rate. It is this channel, which is missing from the current literature, and that turns out to play a very important role on the stability of non- 
fundamental equilibrium exchange rates and the complicated exchange rate behaviour observed in exchange rate markets. We show that the long-run convergence of the fundamental equilibrium exchange rate is related to the macroeconomic fundamental factors and the persistent fluctuations are due to the heterogeneity of the investors. Numerical simulations of the stochastic version of the model show that the model is able to generate the broad features of typical time series behaviour and volatility clustering observed in exchange rate markets.

The paper is organised as follows. In Section 2, we lay out an exchange rate model with nonlinear heterogeneous beliefs in a continuous-time framework. Based on this model, Section 3 analyses the existence of fundamental and non-fundamental steady states. In Section 4, the stability of the fundamental steady state is analysed based on three types of market expectations, those of the fundamentalists, the chartists and then a weighted average of both groups. The conditions and mechanisms under which the exchange rate will deviate from its fundamental value are highlighted. In Section 5, we consider the effect of market noise and Markov switching of the weighting process on the exchange rate market. Some conclusions are given in Section 6. Proofs of some technical results are given in the appendices.

\section{The Model}

The classical Dornbusch (1976) model, which incorporates perfect myopic foresight in predicting exchange rate changes, displays a saddle-point structure. Under the rational expectations assumption of the Dornbusch model, any unanticipated discrete jump in money supply leads to an immediate discrete jump in the exchange rate and the magnitude of this jump is assumed to be precisely that necessary to move the system from its present state onto the stable arm of the saddle point associated with the new equilibrium (see Gray and Turnovsky, 1979 for a very elegant and detailed discussion of this issue). This is the underlying factor behind the well-known overshooting of the exchange rate, which was the important idea introduced by the Dornbusch model.

The model we develop modifies the Dornbusch model by incorporating macroeconomic fundamental factors and the market activity of heterogeneous investors. This means that the market exchange rate is determined not only by the macroeconomic fundamental factors, such as the interest rate differential, but also by the activities of

portfolio managers in the market who aggregate in a certain way the expectations of fundamentalists and chartists. More precisely, consider an exchange rate market populated by many portfolio managers who use a weighted average of the expectations of fundamentalists and chartists as specified below. The following notation is used throughout 
this paper.

$R^{*}$ : the foreign (nominal) rate of interest, taken to be exogenous;

$R$ : the domestic (nominal) interest rate;

$X^{m}, X^{f}, X^{c}$ : the expected rate of exchange depreciation by the portfolio managers,

fundamentalists and chartists, respectively;

$E$ : the logarithm of the current exchange rate, measured in units of

domestic currency per unit of foreign currency;

$P$ : the logarithm of the domestic price level;

$M$ : the logarithm of the domestic nominal money supply, taken to be exogenous;

$Y$ : the logarithm of the domestic real output, taken to be exogenous.

The fundamentalists believe that the depreciation of the spot rate should regress to the fundamental value, which is determined by the interest rate parity condition. Specifically, the expected rate of exchange depreciation of the fundamentalists, $X^{f}$, is assumed to follow

$$
\dot{X}^{f}=\beta_{f}\left(R-R^{*}-X^{f}\right),
$$

where $\beta_{f}>0$ is a constant. Equation (2.1) states that the expectations of the fundamentalists of the rate of exchange depreciation $X^{f}$ form a mean-reverting process to the nominal interest rate differential $R-R^{*}$, with $\beta_{f}$ measuring the speed of adjustment of the mean-reverting process. In the special case where the speed of the adjustment of the fundamentalists is infinite, $\beta_{f}=\infty$, it follows from $\left(1 / \beta_{f}\right) \dot{X}^{f}=R-R^{*}-X^{f}$ that $R-R^{*}=X^{f}$, which is the interest rate parity condition.

In contrast, the chartists use some technical methods to attempt to discover the trend of the market exchange rate. Here we assume that they believe that the change of the log-exchange rate follows a weighted average of past changes of the log-exchange rate ${ }^{1}$, that is

$$
\dot{X}^{c}=\beta_{c}\left(\dot{E}-X^{c}\right)
$$

where $\beta_{c}>0$ measures the speed of adjustment of the chartists to the change of the log-exchange rate or their rate of extrapolation to the log-exchange rate trend. In the special case where the speed of the adjustment of the chartists is infinite, $\beta_{c}=\infty$, it follows from $\left(1 / \beta_{c}\right) \dot{X}^{c}=\left(\dot{E}-X^{c}\right)$ that $X^{c}=\dot{E}$, that is the chartists would have a perfect foresight expectation ${ }^{2}$.

\footnotetext{
${ }^{1}$ If we use $d E(s)$ to represent the instantaneous log-exchange rate change at time $s$ in the continuous time case, we can define $X^{c}(t)=\beta_{c} \int_{-\infty}^{t} e^{-\beta_{c}(t-s)} d E(s)$, which is a declining weighted average of past log-exchange rate changes, then this can be rewritten as (2.2). In the discrete time case, we can define $X_{t+\Delta t}^{c}=\beta_{c}\left(E_{t+\Delta t}-E_{t}\right)+\left(1-\beta_{c}\right) X_{t}^{c}$, which leads to $(2.2)$ in the limit as $\Delta t \rightarrow 0$.

${ }^{2}$ The perfect foresight case is also discussed by Gray and Turnovsky (1979) within the framework of the Dornbusch (1976) model.
} 
The portfolio managers are the investors who actually buy and sell foreign assets in the market. Their decision is based on an aggregate of the expectations of the fundamentalists and chartists of the change in the log-exchange rate. We could think of the portfolio managers as being in a large financial institution that employs groups of fundamental analysts and groups who engage in technical analysis of market data. The role of the managers is to blend these two sources of information in some fashion. We assume that they do this by taking a weighted average of the expectations of the fundamentalists and chartists, so that the expected rate of exchange depreciation of the portfolio managers is given by

$$
X^{m}=H\left((1-\omega) X^{f}+\omega X^{c}\right), \quad \omega \in[0,1],
$$

where $\omega$ and $1-\omega$ represent the market fractions that the portfolio managers attach to the expectations of the chartists and fundamentalists, respectively, and $H(\cdot)$ is a function of the expectations mix that is nonlinear due to the cautiousness of the portfolio managers and is discussed further below.

Exchange Rate Determination - A general model of log-exchange rate adjustment is assumed to be of the form

$$
\dot{E}=X^{m}+Z
$$

where $X^{m}$ is the expected rate of exchange depreciation of the portfolio managers defined by (2.3) and $Z$ represents some macroeconomic fundamental factor, which here is assumed to be determined by the nominal interest rate differential, so that

$$
Z=\beta_{R}\left(R^{*}-R\right)
$$

where $\beta_{R}>0$ is a constant, measuring the impact of the fundamental factor on the exchange depreciation in the market. In order to remain within the spirit of the original Dornbusch model we assume that the interest rate differential is the main driver of exchange rate variation in the macroeconomic fundamental factor. Of course other macro fundamental factors could also be included in $Z$, but we take (2.5) for simplicity.

The first term on the right hand side of (2.4), as in Frankel and Froot (1986), can be thought of as capturing the speculative factors in the market. Here these come from a nonlinear combination of heterogeneous expectations, described by (2.3). The function $H(x)=\mu h(x)$ where $\mu>0$ is a constant scaling factor and $h(\cdot)$ is a nonlinear function satisfying ${ }^{3}$

$$
h(0)=0, h^{\prime}(0)=1, \lim _{x \rightarrow \pm \infty} h^{\prime}(x)=0, \text { and } h^{\prime \prime}(x) x<0 \text { for } x \neq 0 .
$$

Thus the function $H$ is an $S$-shaped function and is illustrated in Fig. 2.1 for different scaling factors. Note that $\mu=H^{\prime}(0)=\max _{x} H^{\prime}(x)$ measures the weight that the portfolio managers put on the expectations of the fundamentalists and chartists when their expected rates of exchange depreciation are near zero.

\footnotetext{
${ }^{3}$ The choice of this functional form is also motivated by the literature (such as Chiarella, Dieci and Gardini, 2002) on heterogeneous agent asset pricing models.
} 
Intuitively, when the deviation of the exchange rate from the fundamental equilibrium increases, the impact of the macroeconomic fundamental factor dominates the bounded nonlinear function in equation (2.4) so that the exchange rate is mean-reverting to the fundamental equilibrium, as will be demonstrated later. The function $H$ captures the cautiousness of the portfolio managers with regard to the speculative elements in the market. That is, portfolio managers are more confident in the aggregate expected rate of depreciation of the fundamentalists and chartists when it is small, and become less confident or more cautious as it becomes larger in absolute value. For example, when the macroeconomic fundamental factor dominates the market, the portfolio managers downplay the aggregate expectation when either the chartists expect a large change by extrapolating strongly the log-exchange rate trend, or when the fundamentalists expect a large exchange rate depreciation from the mean-reverting process to the nominal interest rate differential. However, when the macroeconomic fundamental factor becomes less dominate, the portfolio managers react strongly to the aggregate expectation. The implications and significance of this choice with respect to current exchange rate models will be discussed later in this section.

Relation to Earlier Literature - We now discuss in some detail further motivation behind our model and its relationship to the Dornbusch (1976) model, the Gray and Turnovsky (1979) (GT hereafter) model and the Frankel and Froot (1986) (FF hereafter) model. First, the stabilising mechanism of the macro fundamental factor measured by the interest rate differential in our model is largely motivated by the GT model. To overcome the saddle-point problem of the Dornbusch model and stabilise the exchange rate at its fundamental equilibrium, GT suggest an extension of the perfect myopic foresight mechanism in the Dornbusch model that makes log-exchange rate adjustment sluggish, namely

$$
\dot{E}=\gamma\left[R^{*}-R+X\right]=\gamma X+\gamma\left(R^{*}-R\right)
$$

for some $\gamma>0$. Equation (2.7) should be contrasted with (2.4). Instead of the linear homogeneous market expectation (the first term in (2.7)), we incorporate the nonlinear function $H$ of the expectations of the portfolio managers, due to their cautiousness. It turns out that this extension introduces a mechanism that can generate non-fundamental equilibria.

Second, the rate of change in the spot exchange rate expected by the fundamentalists $X^{f}$ in the FF model is given by $X^{f}=\theta(\bar{E}-E)$, where $\bar{E}$ is the logarithm of the long-run equilibrium exchange rate and $\theta$ is the speed of regression of $E$ to $\bar{E}$. In our model, the rate of exchange depreciation expected by the fundamentalists $X^{f}$ is assumed to follow a mean-reverting process to the nominal interest rate differential $R-R^{*}$, which corresponds to the interest rate parity condition. There are several implications resulting from this difference: (i) The long-run equilibrium $\bar{E}$ in the FF model, which is unknown to the investors in general, is specifically linked to the interest rate differential in our model. 
In some exchange rate markets, there may be no unique long-run equilibrium exchange rate or the exchange rate may shift among different equilibrium levels from time to time because of changing economic conditions, in particular interest rates. (ii) Equation (2.1) describes a sluggish adjustment in the rate of exchange depreciation expected by the fundamentalists. If there were no chartists and the speed of the adjustment of the fundamentalists were infinite, then as we have shown equation (2.1) would lead to the well-known interest rate parity condition assumed in the Dornbusch model.

Third, the exchange rate expected by the chartists in the FF model is assumed to follow a random walk, which is equivalent to assuming that the rate of exchange depreciation expected by the chartists is zero, that is $X^{c}=0$, rather than the conventional assumption that the chartists form their expectations from historical exchange rates. Instead, in our model, the expected depreciation rate by the chartists is a geometrically weighted average of the historical changes in the log-exchange rate with higher weights on the more recent changes. Intuitively, this is consistent with what we observe in financial market practice and survey literature in which geometrically weighted trends are used by practitioners (see, for example the survey by Chiarella, Dieci and He (2009)).

Fourth, in the FF model, in order to characterise the temporary "bubble" in the exchange rate, the weight that the portfolio managers put on the fundamentalist expectation is assumed to be time-dependent and so is written ${ }^{4} \varpi_{t}$ and evolves according to $\Delta \varpi_{t}=\delta\left(\hat{\varpi}_{t-1}-\varpi_{t-1}\right)$, where $0 \leq \delta \leq 1$ measures the speed of adaptation and $\hat{\varpi}_{t-1}$ is defined as the weight given to the fundamentalist view, computed ex post, that would have perfectly predicted the realised change in the previous period. By assuming a random walk process for the exchange rate expected by the chartists, $\hat{\varpi}_{t-1}$ is given by the equation $\Delta E_{t}=\hat{\varpi}_{t-1} \theta\left(\bar{E}-E_{t-1}\right)$. Hence the change of weights that the portfolio managers give to the fundamentalists' view is determined by $\Delta \varpi_{t}=\delta\left[\Delta E_{t} /\left(\theta\left(\bar{E}-E_{t-1}\right)\right)-\varpi_{t-1}\right]$. This specification is based on the assumption of the knowledge of the long-run equilibrium of log-exchange rate $\bar{E}$. As we have discussed in the previous point, when $\bar{E}$ is unknown to the investors, or there is no long-run equilibrium, or the exchange rate shifts among several different equilibrium levels from time to time, choosing $\hat{\varpi}_{t-1}$ as the weight that would have perfectly predicted the realised change in the spot rate would be difficult, if not impossible. In our model, by choosing the weight $\varpi$ as a parameter, we examine how changes in $\varpi$ lead to different exchange rate dynamics ${ }^{5}$. For the deterministic model, the parameter $\varpi$ is a constant and its effect on the exchange rate dynamics will be examined by stability and bifurcation theory in Section 3 and Section 4 . For the stochastic version of the model, it is a random variable and its effect will be analysed by

\footnotetext{
${ }^{4}$ In our model, we use $1-\omega$ to represent the market fraction of the fundamentalists in place of the $\varpi_{t}$ of the FF model.

${ }^{5}$ Ideally, in line with what is done in the recent heterogeneous agent asset pricing literature, the weight should be time-dependent $\left(\varpi_{t}\right)$ and the market would have a systematic way to determine $\varpi_{t}$ based on a certain fitness function. We refer to De Grauwe and Grimaldi (2005a, 2005b, 2006) for a discussion of heterogeneous agent exchange rate models in this spirit within the discrete-time framework.
} 
simulation in Section 5.

Fifth, in the FF model, the log-exchange rate itself is assumed to follow $E_{t}=c \varpi_{t} X^{f}+$ $Z_{t}$, where $c$ is the semi-elasticity of money demand with respect to the alternative rate of return and $Z_{t}$ represents other contemporaneous determinants, which is defined by $Z_{t}=\left(D_{t}-F_{t}\right)-b\left(R_{t}-R^{*}\right)$, where $D_{t}$ and $F_{t}$ are the log of supply of domestic and foreign assets, respectively. By assuming that the actual rate of depreciation is given by

$$
\dot{E}_{t}=\nu\left(\bar{E}-E_{t}\right)
$$

with some constant $\nu>0$, a two-dimensional differential equation system for $\left(E_{t}, \varpi_{t}\right)$ is constructed in the FF model. In our model, rather than assuming (2.8), we model the change in the log-exchange rate $\dot{E}(t)$ directly by assuming that it follows (2.4). This approach has the following advantages: (i) When the log-exchange rate is solely driven by the expected depreciation rate, we would have $\dot{E}=X^{m}$, leading to perfect myopic foresight in predicting log-exchange rate changes. In addition, if there were no chartists, the speed of adjustment of the fundamentalists were infinite and the function $H$ were linear, then we would obtain $\dot{E}=X^{m}=X^{f}=R-R^{*}$, which corresponds to the interest rate parity condition. (ii) When the log-exchange rate is solely driven by the macro fundamental factor $Z(t)$, which depends on the interest rate differential $R-R^{*}$, the nominal interest rate differential should be positively related to the appreciation of the exchange rate. This is because if $R>R^{*}$, the domestic interest rate exceeds the foreign one, there will be a tendency for capital inflow, leading to a depreciation of the foreign currency. The adjustment is reversed if $R<R^{*}$. As we will see from the discussion in the following section, the combination of the speculative and fundamental factors in (2.4) can essentially provide a mechanism that generates multiple equilibrium exchange rates under certain conditions as well as an endogenous mechanism for fluctuation between them.

The Complete Model-In summary, substituting (2.3) and (2.5) into (2.4), the dynamics of the spot log-exchange rate are determined by

$$
\dot{E}=H\left((1-\omega) X^{f}+\omega X^{c}\right)+\beta_{R}\left(R^{*}-R\right) .
$$

The domestic interest rate is determined by the nominal quantity of money, the real income and the goods price via ${ }^{6}$

$$
\alpha_{1} Y-\alpha_{2} R=M-P, \quad \text { where } \quad \alpha_{1}, \alpha_{2}>0
$$

However, for the goods price, we adopt the specification in the original Dornbusch model and assume that it follows a sluggish adjustment process according to which the rate of the log-domestic price adjusts to excess demand. The excess demand is assumed to be a

\footnotetext{
${ }^{6}$ As in the Dornbusch model, (2.10) is obtained by taking the logarithm of the money market equilibrium condition $\mathbb{M} / \mathbb{P}=\mathbb{Y}^{\alpha_{1}} \exp \left(-\alpha_{2} R\right)$, where $\mathbb{M}=\exp (M), \mathbb{P}=\exp (P)$ and $\mathbb{Y}=\exp (Y)$.
} 
decreasing function of the output and domestic nominal interest rate and an increasing function of the relative price $E-P$. More specifically,

$$
\dot{P}=\rho\left[\beta_{0}-\beta_{1} Y-\beta_{2} R+\beta_{3}(E-P)\right],
$$

where $\rho>0, \beta_{1} \in(0,1), \beta_{2}>0, \beta_{3}>0$ and $\beta_{0}$ is a shift parameter.

To sum up, the model of exchange rate, price and the heterogeneous expectations becomes

$$
\left\{\begin{array}{l}
\dot{E}=H\left((1-\omega) X^{f}+\omega X^{c}\right)+\beta_{R}\left(R^{*}-R\right), \\
\dot{P}=\rho\left[\beta_{0}-\beta_{1} Y-\beta_{2} R+\beta_{3}(E-P)\right], \\
\dot{X}^{f}=\beta_{f}\left(R-R^{*}-X^{f}\right), \\
\dot{X}^{c}=\beta_{c}\left(\dot{E}-X^{c}\right),
\end{array}\right.
$$

with $R=\left(\alpha_{1} Y-M+P\right) / \alpha_{2}$ and $R^{*}$ is given. With a constant $R^{*}$, system $(2.12)$ is a 4-dimensional deterministic dynamical system.

Several models in the literature can be recovered from the model (2.12) under the assumption that the rate of exchange depreciation function expected by the portfolio managers is linear, instead of being nonlinear. First, the classical Dornbusch model can be recovered if, under the conditions $\mu=H^{\prime}(0)=\beta_{R}+1$ and $H(x)=\mu x$, we assume either $\omega=0$ (zero weight on the chartist expectation) and $\beta_{f}=\infty$ (the speed of adjustment of the fundamentalists becomes infinite), or $\omega=1$ (zero weight on the fundamentalist expectation) and $\beta_{c}=\infty$ (the chartists extrapolate infinitely rapidly). Second, by choosing $\omega=1, \beta_{c}=\infty, H(x)=\gamma x$ and $\beta_{R}=\gamma$, our model (2.12) yields the Gray and Turnovsky (1979) model with sluggish adjustment in the exchange rate.

In the following two sections, we examine the existence of multiple equilibria and their stability. By introducing market noise and a Markov switching process into the weighting parameter of the heterogeneous expectations, we establish a stochastic version of the deterministic system (2.12) in Section 5. The analysis of the dynamics of the deterministic system provides some insight into the dynamics of the stochastic model.

\section{Existence of Multiple Equilibria}

The evolution of the system (2.12) describing the dynamics of the log-exchange rate depends on the characteristics of its steady states, which encapsulate the long-run behaviour of the system. In this section, we examine the existence of equilibria. In particular, we provide conditions for the existence of multiple steady states. The long-run behaviour of the system will be analysed in the following section.

Let $\mathcal{S}:=\left(\bar{E}, \bar{P}, \bar{X}^{f}, \bar{X}^{c}\right)$ be a steady state of the system (2.12). Then $\mathcal{S}$ satisfies

$$
\left\{\begin{aligned}
\bar{E} & =\frac{1}{\beta_{3}}\left[-\beta_{0}+\beta_{1} Y+\beta_{2} \bar{R}+\beta_{3} \bar{P}\right], \\
\bar{P} & =M-\alpha_{1} Y+\alpha_{2}\left(R^{*}+\bar{X}^{f}\right), \\
\beta_{R} \bar{X}^{f} & =H\left((1-\omega) \bar{X}^{f}\right), \\
\bar{X}^{c} & =0
\end{aligned}\right.
$$


where $\bar{R}=R^{*}+\bar{X}^{f}$ satisfies the interest rate parity condition. Obviously $\bar{X}^{f}=0$ is always a solution of (3.1c). In this case, the corresponding steady state is given by $\mathcal{S}_{0}:=\left(E^{*}, P^{*}, 0,0\right)$ and the zero interest rate parity condition holds, that is $\bar{R}-R^{*}=0$, where $E^{*}$ and $P^{*}$ are defined in (3.2) below. This steady state corresponds to the classical equilibrium of the exchange rate in the Dornbusch (1976) model and hence we call $\mathcal{S}_{0}=\left(E^{*}, P^{*}, 0,0\right)$ the fundamental steady state.

Figure 3.1 here

When $\mu(1-\omega)>\beta_{R}$, equation (3.1c) has two other solutions, $\bar{X}^{f}=X_{ \pm}^{*}$, satisfying $X_{-}^{*}<0<X_{+}^{*}$, the graphical determination of which is illustrated in Figure 3.1. The condition $\mu(1-\omega)>\beta_{R}$ can be interpreted as either the portfolio managers giving a higher weight (relatively) to the expectations of the fundamentalists, or that they are having a greater impact relative to the macro-environment factor. In this case, the system (2.12) has both the fundamental equilibrium and other non-fundamental equilibria. At $\bar{X}^{f}=X_{ \pm}^{*}$, the non-zero interest rate parity condition holds, that is $X_{ \pm}^{*}=\bar{R}_{ \pm}-R^{*} \neq 0$. At the same time, the steady states corresponding to $\bar{X}^{f}=X_{ \pm}^{*}$ are given by $\mathcal{S}_{ \pm}:=\left(\bar{E}, \bar{P}, \bar{X}^{f}, \bar{X}^{c}\right)=\left(\bar{E}_{ \pm}, \bar{P}_{ \pm}, X_{ \pm}^{*}, 0\right)$ with $\bar{E}_{ \pm}=E^{*}+\left(\frac{\beta_{2}}{\beta_{3}}+\alpha_{2}\right) X_{ \pm}^{*}$ and $\bar{P}_{ \pm}=P^{*}+\alpha_{2} X_{ \pm}^{*}$. Because of the deviation of the log-exchange rate from the fundamental level $E^{*}$ and the corresponding interest rate differential away from zero, we call $\mathcal{S}_{ \pm}$nonfundamental steady-states of the system. Summarising the above analysis, we obtain the following result on the existence of steady states.

Theorem 3.1 For given $R^{*}$, let

$$
P^{*}=M-\alpha_{1} Y+\alpha_{2} R^{*}, \quad E^{*}=\frac{1}{\beta_{3}}\left[-\beta_{0}+\beta_{1} Y+\beta_{2} R^{*}+\beta_{3} P^{*}\right] .
$$

Then

(1) the fundamental steady state $\mathcal{S}_{0}=\left(E^{*}, P^{*}, 0,0\right)$ is always a steady state of the system (2.12);

(2) if $\mu(1-\omega)>\beta_{R}$, then the system (2.12) has another two non-fundamental steady states $\mathcal{S}_{ \pm}=\left(\bar{E}_{ \pm}, \bar{P}_{ \pm}, \bar{X}_{ \pm}^{f}, \bar{X}_{ \pm}^{c}\right)=\left(E^{*}+\left(\alpha_{2}+\beta_{2} / \beta_{3}\right) X_{ \pm}^{*}, P^{*}+\alpha_{2} X_{ \pm}^{*}, X_{ \pm}^{*}, 0\right)$ in which $X_{ \pm}^{*}$ are the non-zero roots of $\beta_{R} X^{*}=H\left((1-w) X^{*}\right)$.

It is interesting to see that the condition for the existence of the non-fundamental steady states is independent of the adjustment rate of the expectations of the fundamentalists and chartists, as measured by $\beta_{f}$ and $\beta_{c}$ respectively. Rather the condition depends on the relation among the strength of the portfolio managers' response $(\mu)$, the weight ascribed to the fundamentalists $(1-\omega)$ and the strength of the macroeconomic fundamental factor $\left(\beta_{R}\right)$. Note that both $\bar{P}_{ \pm}$and $\bar{E}_{ \pm}$increase as $X_{ \pm}^{*}$ increases. The levels of $X_{ \pm}^{*}$ depend on not only $\mu$ and $\omega$, but also on $\beta_{R}$. As $\beta_{R}$ increases, $X_{ \pm}^{*}$ and 
hence the non-fundamental equilibrium levels decrease. Note that when $\beta_{R}$ increases to $\mu(1-\omega), X_{ \pm}^{*}$ converge to 0 and the corresponding two non-fundamental steady states $\mathcal{S}_{ \pm}$converge to the fundamental steady state $\mathcal{S}_{0}$. In contrast, an increase in $\mu$ leads the non-fundamental steady states $\mathcal{S}_{ \pm}$to move further away from the fundamental steady state $\mathcal{S}_{0}$. More discussion about the economic intuition of Theorem 3.1 will be given in the following section.

\section{Stability of the Exchange Rate Market}

To better understand the dynamic evolution of the system (2.12), we need to analyse the local stability and bifurcations of the fundamental equilibrium $\mathcal{S}_{0}=\left(E^{*}, P^{*}, 0,0\right)$, which is characterised by the following result.

Theorem 4.1 For the system (2.12), the fundamental steady state $\mathcal{S}_{0}$ is locally asymptotically stable (LAS) if and only if

$$
d_{1}>0, \quad d_{2}>0, \quad d_{4}>0, \quad \Delta_{4}>0,
$$

where

$$
\begin{aligned}
d_{1} & :=\beta_{c}(1-\mu \omega)+\beta_{f}+\beta_{p} \\
d_{2} & :=\beta_{c}(1-\mu \omega)\left(\beta_{f}+\beta_{p}\right)+\beta_{p} \beta_{f}+\beta_{e} \beta_{R} \\
d_{3} & :=\beta_{p} \beta_{c} \beta_{f}(1-\mu \omega)+\beta_{e} \beta_{R} \beta_{c}+\beta_{e} \beta_{f}\left(\beta_{R}-\mu+\mu \omega\right), \\
d_{4} & :=\beta_{e} \beta_{c} \beta_{f}\left(\beta_{R}-\mu+\mu \omega\right) \\
\Delta_{4} & :=d_{1} d_{2} d_{3}-d_{1}^{2} d_{4}-d_{3}^{2}
\end{aligned}
$$

with

$$
\beta_{p}:=\rho\left(\beta_{3}+\beta_{2} / \alpha_{2}\right)(>0), \quad \beta_{e}:=\rho \beta_{3} / \alpha_{2}(>0) .
$$

The conditions (4.1) are obtained by application of the Routh-Hurwitz Theorem to the dynamical system (2.12) as explained in Appendix 1. When the conditions (4.1) are not satisfied, the fundamental steady state $\mathcal{S}_{0}$ becomes unstable and the system $(2.12)$ exhibits complex dynamics through different types of bifurcations. In particular, on the manifold $d_{4}=0$, there is at least one zero eigenvalue. At $d_{4} \lesssim 0$, it follows from Theorem 3.1 that the system $(2.12)$ has two additional non-fundamental steady states $\mathcal{S}_{ \pm}$. Hence at $d_{4} \lesssim 0$, the system (2.12) diverges from the fundamental steady state and numerical simulations show that the system converges locally to one of the non-fundamental steady states $\mathcal{S}_{ \pm}$, depending on the initial conditions. In nonlinear dynamical systems theory, see Kuznetsov (2004), this phenomenon is referred to as a pitchfork bifurcation and consequently $d_{4}=0$ is called a pitchfork boundary. On the manifold $\Delta_{4}=0$, there is a pair of conjugate imaginary eigenvalues. This means that the fundamental steady state $\mathcal{S}_{0}$ loses its stability and, in its neighborhood, a stable limit cycle can emerge. This 
phenomenon is referred to as a Hopf bifurcation and consequently $\Delta_{4}$ is called a Hopf manifold or Hopf boundary.

Given the complexity of the stability conditions and the difficulty of saying very much in the general case, we consider three special cases in detail in the following in order to gain some insights into the dynamical behaviour of the general case. These three cases help us to understand the different roles played by the different expectation mechanisms on the dynamics of the system (2.12), including the stability and dynamical behaviour resulting from the instability of the fundamental equilibrium. The portfolio managers put all the weight on either the fundamentalist or the chartist expectations in the first two cases, or on both in the third case. The first two cases include the classical Dornbusch (1976) model and the Gray and Turnovsky (1979) model as special situations. The analysis in the first two special cases provides some helpful insight on the understanding of the third general case.

In the subsequent analysis, we shall illustrate many of our points with numerical simulations, for which, unless stated otherwise, we take the parameter set in Table 1 and set $H(x)=\mu h(x)$ where $h(x)=\tanh (\gamma x) / \gamma$ and the other parameters will be specified later.

\begin{tabular}{ccccccccccc}
\hline$M$ & $Y$ & $R^{*}$ & $\rho$ & $\beta_{0}$ & $\beta_{1}$ & $\beta_{2}$ & $\beta_{3}$ & $\alpha_{1}$ & $\alpha_{2}$ & $\gamma$ \\
\hline 3.5 & 2 & 0.01 & 0.25 & 2.1 & 0.5 & 1 & 0.5 & 0.5 & 4 & 10 \\
\hline
\end{tabular}

Table 1: Parameter values

\subsection{The model with fundamentalist expectations}

We first consider the case of $\omega=0$, that is the portfolio managers put all the weight on the expectations of the fundamentalists. In this case, the system (2.12) reduces to the three-dimensional system

$$
\left\{\begin{aligned}
\dot{E} & =H\left(X^{f}\right)+\beta_{R}\left(R^{*}-\frac{\alpha_{1} Y-M}{\alpha_{2}}-\frac{P}{\alpha_{2}}\right), \\
\dot{P} & =\rho\left[\beta_{0}-\beta_{1} Y-\beta_{2}\left(\frac{\alpha_{1} Y-M}{\alpha_{2}}+\frac{P}{\alpha_{2}}\right)+\beta_{3}(E-P)\right], \\
\dot{X}^{f} & =\beta_{f}\left(\frac{\alpha_{1} Y-M}{\alpha_{2}}+\frac{P}{\alpha_{2}}-R^{*}-X^{f}\right) .
\end{aligned}\right.
$$

Concerning the dynamical behaviour of the system (4.4), we obtain the following result.

Theorem 4.2 For the system (4.4),

(i) if $\beta_{R}>\mu$, then the fundamental steady state $\mathcal{S}_{0}=\left(E^{*}, P^{*}, 0\right)$ of (4.4) is LAS;

(ii) at $\beta_{R}=\mu$, the fundamental steady state $\mathcal{S}_{0}$ undergoes a pitchfork bifurcation;

(iii) if $\beta_{R}<\mu$, then the fundamental steady state $\mathcal{S}_{0}$ is unstable and the two nonfundamental steady states $\mathcal{S}_{ \pm}=\left(E^{*}+\left(\frac{\beta_{2}}{\beta_{3}}+\alpha_{2}\right) X_{ \pm}^{*}, P^{*}+\alpha_{2} X_{ \pm}^{*}, X_{ \pm}^{*}\right)$ of $(4.4)$ exist and are $L A S$, where $X_{ \pm}^{*}$ satisfy $H\left(X_{ \pm}^{*}\right)=\beta_{R} X_{ \pm}^{*}$ and $X_{ \pm}^{*} \neq 0$. 
Figure 4.1 here

The proof of Theorem 4.2 is given in Appendix 1. The stability/instability of the fundamental equilibrium $\mathcal{S}_{0}$ and its pitchfork bifurcation boundary are plotted in Figure 4.1 in $\left(\mu, \beta_{R}\right)$ parameter space. The economic implications of Theorem 4.2 are far more significant than are apparent at first glance, and we elaborate on them in the following discussion.

Figure 4.2 here

First, the stabilising effect of the fundamental equilibrium due to the sluggish exchange rate adjustment considered in Gray and Turnovsky (1979) is also present in our model. As we have mentioned earlier, when $\beta_{f}=\infty$, the expected depreciation rate by the fundamentalists $X^{f}$ in (4.4) is equal to the nominal interest rate differential $X^{f}=R-R^{*}$ and consequently (4.4) simplifies to the two-dimensional system

$$
\left\{\begin{array}{l}
\dot{E}=H\left(R-R^{*}\right)+\beta_{R}\left(R^{*}-R\right), \\
\dot{P}=\rho\left[\beta_{0}-\beta_{1} Y-\beta_{2} R+\beta_{3}(E-P)\right],
\end{array}\right.
$$

with $R=\left(\alpha_{1} Y-M+P\right) / \alpha_{2}$. The characteristic polynomial of the fundamental steady state equilibrium $\left(E^{*}, P^{*}\right)$ is given by $p(\lambda)=\lambda^{2}+\beta_{p} \lambda+\beta_{e}\left(\beta_{R}-\mu\right)$ and the corresponding two eigenvalues $\lambda_{1}$ and $\lambda_{2}$ satisfy $\lambda_{1}+\lambda_{2}=-\beta_{p}<0$ and $\lambda_{1} \lambda_{2}=\beta_{e}\left(\beta_{R}-\mu\right)$. So when the macro fundamental factor dominates the speculative factor due to the expectations of the fundamentalists in the sense that $\beta_{R}>\mu$, the fundamental steady state equilibrium $\left(E^{*}, P^{*}\right)$ is stable as illustrated in Figure $4.2(\mathrm{a})$.

Second, when the macro fundamental factor is dominated by the speculative factor of the expectations of the fundamentalists, that is $\beta_{R}<\mu$, the fundamental equilibrium $\left(E^{*}, P^{*}\right)$ becomes a saddle point, see Figure $4.2(\mathrm{c})$, which corresponds to the Dornbusch saddle-point case discussed in Gray and Turnovsky (1979). In particular, if we take $\mu=\beta_{R}+1\left(>\beta_{R}\right)$ and $H(x)=\mu x$, then (4.5) reduces to

$$
\left\{\begin{array}{l}
\dot{E}=R-R^{*}, \\
\dot{P}=\rho\left[\beta_{0}-\beta_{1} Y-\beta_{2} R+\beta_{3}(E-P)\right],
\end{array}\right.
$$

which is the classical Dornbusch model. However due to the introduction of the nonlinear $S$-shaped expectation function for the portfolio managers, the saddle-point instability of the fundamental equilibrium leads the exchange rate to stabilise at one of the two non-fundamental equilibria, depending on the initial market conditions, as illustrated in Figure 4.2(b). Thus, the emergence of the two non-fundamental steady-states avoids the explosive outcome for the dynamics implied by the saddle-point instability without imposing the instantaneous (and unexplained) jump in the exchange rate to place the system on a stable arm of the saddle. 
The economic intuition of the above implications is as follows. Consider the special case with $\beta_{R} \rightarrow 0$ in (4.5) (so that $\beta_{R}<\mu$ ). When $X^{f}=R-R^{*}>0$, the rate of exchange depreciation expected by the fundamentalists is positive, leading to a higher log-exchange rate $E$ and hence a higher log-price $P$, which in turn pushes the expected rate of exchange depreciation to be even higher. Consequently, the fundamental steady state becomes unstable. However, since the portfolio managers become more cautious as the expected rate of exchange depreciation becomes larger (reflected in their nonlinear $S$-shaped function $H$ of the expected depreciation rate), the system does not explode but rather converges to one of the two non-fundamental steady state equilibria $\mathcal{S}_{ \pm}=$ $\left(\bar{E}_{ \pm}, \bar{P}_{ \pm}\right)=\left(E^{*}+\left(\frac{\beta_{2}}{\beta_{3}}+\alpha_{2}\right) X_{ \pm}^{*}, P^{*}+\alpha_{2} X_{ \pm}^{*}\right)$ where $X_{ \pm}^{*}$ satisfy the non-zero interest rate parity condition, that is $X_{ \pm}^{*}=\bar{R}_{ \pm}-R^{*} \neq 0$, and $H\left(X_{ \pm}^{*}\right)=\beta_{R} X_{ \pm}^{*}$.

Figure 4.3 here

For the general case with $\beta_{f}>0$, phenomena similar to those displayed in Figure 4.2 occur, and the appearance of two locally stable non-fundamental steady state equilibria corresponds to the occurrence of a pitchfork bifurcation at the fundamental equilibrium. Figure 4.3(a) shows a bifurcation plot of the log-exchange rate with respect to the parameter $\beta_{R}$ for $\beta_{f}=0.8$. For the given value $\mu=1$, it shows that a pitchfork bifurcation occurs at $\beta_{R}=\mu=1$. For $\beta_{R}>\mu$, the fundamental steady state is stable, but for $\beta_{R}<\mu$, it is unstable and two locally stable non-fundamental steady states appear. Figure 4.3(b) shows the dynamics for the log-exchange rate when $\beta_{R}=0.85<\mu=1$. For different initial values for $E_{0}$, it shows that $E \rightarrow \bar{E}_{+}$or $E \rightarrow \bar{E}_{-}$as time increases.

In summary, the Dornbusch model and its various extensions have played an important role in the development of the exchange rate literature. It is very interesting to see how the simple model (4.4) retains the basic idea of the Dornbusch model and can also generate a number of new desirable features. The original Dornbusch model had some notable shortcomings. First, the stability of exchange rate markets is achievable only when agents adopt rational expectations so there is no room for the bounded rationality uncovered in the survey literature cited earlier. Also, speculative activity, which plays a very important role (in fact it is a much bigger component of exchange rate transactions than those based on "real" factors) in exchange rate markets, is missing. In the model presented here, this stability can be achieved as a result of interaction of speculation and the macro fundamental factor. Second, if we do believe that in exchange rate markets there exist steady state equilibria, our result shows that there may exist multiple stable equilibria. Depending on the market conditions, the exchange rate can stabilise at different steady state equilibrium levels and changing conditions can cause the exchange rate to shift among different equilibrium levels. Third, interest rate parity holds at all the three steady state equilibria in our model, even when the interest rate differential $R-R^{*}$ is not zero. In the Dornbusch model, the unique steady state in which the interest rate parity condition holds is the one with no interest rate differential. As far as we are 
aware, this is the first result on the dynamics of the interest rate parity condition that allows the interest rate differential to be nonzero.

\subsection{The model with the chartist expectation}

We now consider the second special case of $\omega=1$. That is, the expectations of the portfolio managers depend solely on the expectations of the chartists, which follow a sluggish adjustment to a geometric average of the historical changes in the log-exchange rate. In this case, the system (2.12) reduces to the 3-dimensional system

$$
\left\{\begin{array}{l}
\dot{E}=H\left(X^{c}\right)+\beta_{R}\left(R^{*}-\frac{\alpha_{1} Y-M}{\alpha_{2}}-\frac{P}{\alpha_{2}}\right), \\
\left.\dot{P}=\rho\left[\beta_{0}-\beta_{1} Y-\beta_{2}\left(\frac{\alpha_{1} Y-M}{\alpha_{2}}\right)+\beta_{3} E-\left(\frac{\beta_{2}}{\alpha_{2}}+\beta_{3}\right) P\right)\right], \\
\dot{X}^{c}=\beta_{c}\left(H\left(X^{c}\right)+\beta_{R}\left(R^{*}-\frac{\alpha_{1} Y-M}{\alpha_{2}}-\frac{P}{\alpha_{2}}\right)-X^{c}\right) .
\end{array}\right.
$$

Since $\omega=1$ and $\beta_{R}>0$, the condition $(1-\omega) \mu>\beta_{R}$ is not satisfied in this case. It then follows from Theorem 3.1 that the fundamental equilibrium $\mathcal{S}_{0}=\left(\bar{E}, \bar{P}, \bar{X}^{c}\right)=$ $\left(E^{*}, P^{*}, 0\right)$ is the unique steady state of the system (4.7) at which $\bar{R}=R^{*}$. With regards to the stability of the steady state and its bifurcation, we have the following result.

Theorem 4.3 For the system (4.7), let

$$
\beta_{R}^{*}:=\frac{\beta_{p}^{2}(1-\mu)(2-\mu+2 \sqrt{1-\mu})}{\mu^{2} \beta_{e}}, \quad \beta_{c}^{ \pm}:=\frac{-b_{2} \pm \sqrt{b_{2}^{2}-4 b_{1} b_{3}}}{2 b_{1}},
$$

where $b_{1}:=\beta_{p}(1-\mu)^{2}, b_{2}:=\beta_{p}^{2}(1-\mu)-\beta_{e} \mu \beta_{R}$ and $b_{3}:=\beta_{e} \beta_{p} \beta_{R}$.

(i) For $0<\mu<1$,

$-\mathcal{S}_{0}$ is LAS if either (i) $\beta_{R}<\beta_{R}^{*}$, or (ii) $\beta_{R}>\beta_{R}^{*}$ and $0<\beta_{c}<\beta_{c}^{-}$, or (iii) $\beta_{R}>\beta_{R}^{*}$ and $\beta_{c}>\beta_{c}^{+}$;

$-\mathcal{S}_{0}$ is unstable for $\beta_{R}>\beta_{R}^{*}$ and $\beta_{c}^{-}<\beta_{c}<\beta_{c}^{+}$;

- if $\beta_{R}>\beta_{R}^{*}, \mathcal{S}_{0}$ undergoes Hopf bifurcations at $\beta_{c}=\beta_{c}^{ \pm}$;

(ii) For $\mu=1, \mathcal{S}_{0}$ is LAS when $0<\beta_{c}<\beta_{p}$, unstable when $\beta_{c}>\beta_{p}$, and undergoes a Hopf bifurcation at $\beta_{c}=\beta_{p}$.

(iii) For $\mu>1, \mathcal{S}_{0}$ is LAS when $0<\beta_{c}<\beta_{c}^{-}$, unstable when $\beta_{c}>\beta_{c}^{-}$, and undergoes a Hopf bifurcation at $\beta_{c}=\beta_{c}^{-}$.

Depending on the balance between the macro fundamental factor and the chartist expectations, Theorem 4.3 provides a very interesting result about the stability of the 
fundamental steady state $\mathcal{S}_{0}$ and periodic oscillations ${ }^{7}$ of the market around the steady state when it becomes unstable. In the following discussion, we focus on the economic intuition of the different possible situations.

For the case of $\mu=1$, from Theorem 4.3(ii), the stability of $\mathcal{S}_{0}$ depends on the balance between $\beta_{c}$ and $\beta_{p}$. Given that $\beta_{p}=\rho\left(\beta_{2} / \alpha_{2}+\beta_{3}\right)>0$ (see (4.3)), it follows from (4.7b) that $\beta_{p}$ measures the speed of the convergence of the log-price level $P$ to an equilibrium. Obviously, $\beta_{c}$ measures the speed of the convergence of the expected depreciation rate of the chartists $X^{c}$ to the instantaneous depreciation rate $\dot{E}$ in the market, or the weight on the latest change in the log-exchange rate when forming the expected depreciation rate by the chartists. Intuitively, on the one hand, when $\beta_{p}>\beta_{c}$, that is, the speed of the convergence of the log-price $P$ is faster than that of $X^{c}$, the extrapolation of the expected depreciation rate from the chartists is weak and its impact is not so significant. Consequently, the market converges to its fundamental steady state. On the other hand, when $\beta_{p}<\beta_{c}$, that is, the speed of the convergence of the log-price $P$ is slower than that of $X^{c}$, any change in the exchange rate and the price is extrapolated quickly by the chartists, which in turn feeds back into the market and generates instability. For instance, if the initial values $(E, P)$ are above the steady state levels $\left(E^{*}, P^{*}\right)$ and $X^{c}$ starts near zero, the system leads to a high interest rate $R$. For given $R^{*}$, this leads to a negative change in the log-exchange rate. Then the extrapolation of the expected depreciation from the chartists will very quickly push the log-exchange rate even lower and bring it further below $E^{*}$. This low log-exchange rate will push the log-price $P$ down below $P^{*}$. Because of the slow convergence in the log-price $P$, this trend will continue and the log-exchange rate will continue to drift down below $E^{*}$ until the moment at which the impact of the extrapolation from the chartists becomes less significant for large $X^{c}$ due to the cautious reaction of the portfolio managers (reflected in their $S$ shaped extrapolation function). Then the price moves back and a reverse cycle starts. Consequently, the fundamental steady state becomes unstable and a periodic fluctuation around the steady state appears.

Figure 4.4 here

For the case of $\mu>1$, a similar intuition applies. The only difference here is that, because of higher weight $\mu$ from the expectations of the portfolio managers, the impact of the extrapolation from the chartists becomes even stronger. To maintain the stability of the fundamental steady state, a lower extrapolation coefficient $\beta_{c}$ from the chartists is required to be bounded above by $\beta_{c}^{-}$satisfying $\beta_{c}^{-}<\beta_{p}$. For $\mu \geq 1$, Figure 4.4(a) shows the bifurcation of the log-exchange rate $E$ in terms of the parameter $\beta_{c}$, which clearly

\footnotetext{
${ }^{7}$ The stability of the periodic solution induced by the Hopf bifurcation depends on the nonlinearity of the $H$ function. It is difficult to obtain precise mathematical conditions for our model, but numerical simulations indicate that the periodic solution is stable, as demonstrated in the bifurcation plots in Figure 4.4.
} 
indicates the stability of the fundamental steady state for the log-exchange rate when $\beta_{c}$ is small and the stable periodic orbits induced by the Hopf bifurcation when $\beta_{c}$ is large.

For the case of $\mu<1$, the economic intuition we can obtain from our results seems very limited. In general, the fundamental coefficient $\beta_{R}$ plays a stabilising role while the extrapolation activity on the expected depreciation rate from the chartists destabilises the market. For example, in the cases of $\beta_{R}<\beta_{R}^{*}$, and $\beta_{R}>\beta_{R}^{*}$ and $\beta_{c}<\beta_{c}^{-}$, the fundamental steady state is stable. Note that when $\beta_{R}>\beta_{R}^{*}$, a balance between the macro fundamental factor $\left(\beta_{R}\right)$ and the extrapolation of the chartists $\left(\beta_{c}\right)$, is necessary to achieve the stability of the fundamental steady state, and this is the source of the condition $\beta_{c}<\beta_{c}^{-}$. For the case that $\beta_{R}>\beta_{R}^{*}$ and $\beta_{c}^{-}<\beta_{c}<\beta_{c}^{+}$, a strong tendency to convergence (measured by $\beta_{R}$ ) could generate a trend which would be extrapolated by the chartists (measured by $\beta_{c}$ ), pushing the market from one extreme to the other, even though the market does not react actively to the chartist expectation (since $\mu<1$ ). However, the condition $\beta_{c}>\beta_{c}^{+}$is unexpected and it does not follow this intuition. One possible explanation is that a higher $\beta_{c}$ indicates that more weight is given to the most recent changes, meaning that the chartists move closer to the perfect foresight limit when forming their expectations. This could help to stabilise the fundamental steady state. In any case, our analysis indicates how a simple expectation scheme like the geometrically weighted average of the historical changes in the log-exchange rate can generate very complicated dynamics. For $\mu<1$, Figure 4.4(b) demonstrates the bifurcation of the log-exchange rate $E$ in terms of the parameter $\beta_{c}$, which clearly indicates the stability of the steady state for the log-exchange rate when $\beta_{c}$ is either small or large, and the appearance of stable periodic orbits induced by the Hopf bifurcations when $\beta_{c}$ is in the intermediate region determined by $\beta_{c}^{-}$and $\beta_{c}^{+}$.

The results and discussions for these two special cases illustrate explicitly the different roles played by different expectations of the fundamentalists and chartists and the different ways that the exchange rate dynamics are generated through different types of bifurcations. An understanding of this difference provides insight into the exchange rate dynamics when the expectations of the portfolio managers are formed as a weighted average of the expectations of the fundamentalists and chartists, to which we turn in the following subsection.

\subsection{The model with the expectations of the fundamentalists and chartists}

We now consider the general case when the expectations of the portfolio managers are formed from a weighted average of the heterogeneous expectations of the fundamentalists and chartists. From subsections 4.1 and 4.2 , we have seen that different expectation schemes lead to different market behaviour when the fundamental equilibrium loses its stability. We now examine how such a mixture of the expectation schemes influences 
the market exchange rate. Intuitively, we expect to see the types of behaviour induced by both pitchfork and Hopf bifurcations when the fundamental steady state loses its stability. Depending on the dominance of the two schemes, the system may display one type of behaviour rather than the other type. Indeed our analysis below confirms this intuition. To simplify the mathematical exposition, we consider two simplified cases which shed light on the general case. The first case explores the different dynamics induced by the change of the weighting parameter $\omega$, whilst the second case demonstrates the stability property when the heterogeneous expectations are balanced in a certain sense. We examine the first case in detail here and relegate the second case to the Appendix 2.

In the first case, in order to explore the different dynamics induced by the change of the weighing parameter $\omega$, we assume that the speeds of adjustment of both the fundamentalists and chartists to their beliefs are the same, that is $\beta_{f}=\beta_{c}$, and the intensity of the adjustment of the portfolio managers on their expected depreciation rate is unity, that is $\mu=1$. By an application of Theorem 4.1 we obtain the following theorem that provides a result on the stability of the fundamental equilibrium and market behaviour when it loses its stability.

Theorem 4.4 For the system (2.12), assume $\beta_{p}<\beta_{f}=\beta_{c}$, and $\mu=1$. Let $\phi:=\beta_{f}=$ $\beta_{c}$ and

$$
\omega^{*}=1+\frac{\beta_{p}^{2}+\beta_{e}\left(\beta_{R}+1\right)-\sqrt{\left(\beta_{p}^{2}-\beta_{e}\left(1+\beta_{R}\right)\right)^{2}+4 \beta_{p} \beta_{e}\left(\beta_{p}+\phi \beta_{R}\right)}}{2 \beta_{p} \phi} .
$$

(a) If $\beta_{R} \leq 1$, then $1-\beta_{R}<\omega^{*}<1$ and the fundamental equilibrium $\mathcal{S}_{0}$ (i) is unstable for $0<\omega<1-\beta_{R}$; (ii) undergoes a pitchfork bifurcation at $\omega=1-\beta_{R}$; (iii) is stable for $1-\beta_{R}<\omega<\omega^{*}$; (iv) undergoes a Hopf bifurcation at $\omega=\omega^{*}$; (v) is unstable for $\omega^{*}<\omega<1$.

(b) If $\beta_{R}>1$, then $0<\omega^{*}<1$ and the fundamental equilibrium $\mathcal{S}_{0}$ (i) is stable for $0<\omega<\omega^{*}$; (ii) undergoes a Hopf bifurcation at $\omega=\omega^{*}$; (iii) is unstable for $\omega^{*}<\omega<1$.

It is interesting to see from Theorem 4.4 how changes in the weight $\omega$ lead to different market behaviour. The result confirms the intuition induced from our analysis in the previous two special cases. However, the nature of the exact connection to the two special cases $\omega=0$ and $\omega=1$, as well as the question as to whether a weighted average of the two expectation schemes can stabilise an otherwise unstable fundamental steady state are what we shall be concerned with in the following discussion.

On the one hand, when $\omega=0$ and $\beta_{R}<1=\mu$, it follows from Theorem 4.2 that $\mathcal{S}_{0}$ is unstable and undergoes a pitchfork bifurcation at $\beta_{R}=\mu=1$. By taking the limiting case of $\omega \rightarrow 0$, we have from Theorem 4.4 that a pitchfork bifurcation occurs as $\beta_{R} \rightarrow 1(=\mu)$ and consequently we obtain the exact same result as in the case of $\omega=0$. 
On the other hand, when $\omega=1, \beta_{p}<\phi=\beta_{c}$, it follows from Theorem 4.3(ii) that $\mathcal{S}_{0}$ is unstable, which is implied by Theorem 4.4. In this way, we see how the results of Theorems 4.2 and 4.3 are consistent with those of Theorem 4.4.

Figure 4.5 here

For $0<\omega<1$, the stability and the induced bifurcations based on Theorem 4.4 are illustrated by the bifurcation plot of the log-exchange rate $E$ in terms of the parameter $\omega$ in Figure 4.5 for the indicated set of parameters. Two things are clearly indicated by this figure. First, it shows different types of bifurcations when the market expectation is dominated by either one of the expectation schemes. When $\mathcal{S}_{0}$ loses its stability for $\omega<$ $1-\beta_{R}$, the expectations of the fundamentalists are responsible for the induced pitchfork bifurcation, while the expectations of the chartists are responsible for the induced Hopf bifurcation when $\omega>\omega^{*}$. Second, a mixture of the two expectations can stabilise an otherwise unstable steady state $\mathcal{S}_{0}$ when $\omega \in\left(1-\beta_{R}, \omega^{*}\right)$. This stabilising effect of combining different types of expectations is also shared by asset pricing models with heterogeneous beliefs (see, for example, Chiarella and He, 2002).

Figure 4.6 here

We now extend our analysis on the stabilising effect of the combined expectation schemes further when two of the key parameters $\beta_{R}$ and $\phi$ change. First, recall that the parameter $\beta_{R}$ measures the strength of the macro fundamental factor related to the interest rate differential. Note that the pitchfork boundary $\omega=1-\beta_{R}$ is monotonically decreasing in $\beta_{R}$. This means that a larger $\beta_{R}$ results in a higher weight put by the portfolio managers on the fundamentalist expectation, which in turn can stabilise the fundamental steady state $\mathcal{S}_{0}$. Second, for the Hopf bifurcation boundary $\omega=\omega^{*}$, we can show that $-1<d \omega^{*} / d \beta_{R}<0$ for $\beta_{R}>0$, implying that the Hopf boundary $\omega^{*}$ also decreases as $\beta_{R}$ increases. This is clearly demonstrated in Figure 4.6(a) in which the stability region and bifurcation boundaries are plotted in the $\left(\beta_{R}, \omega\right)$ parameter space. Therefore, as the strength $\beta_{R} \in(0,1]$ of the macro fundamental factor increases, the stability interval $\omega \in\left(1-\beta_{R}, \omega^{*}\right)$ shifts to the left. In other words, the stability region of the fundamental equilibrium in the weighting parameter $\omega$ is enlarged. One can also see that the fundamental steady state can be stabilised by either increasing $\omega$ from below or decreasing $\omega$ from above, meaning a balanced combination of the two expectations can stabilise the fundamental steady state. In addition, for fixed $\beta_{R}>1$, the fundamental steady state is destabilised by increasing $\omega$ further, leading to periodic fluctuations around the steady state induced by a Hopf bifurcation.

The other parameter $\phi=\beta_{f}=\beta_{c}$ measures the speed of adjustment of the expectations of the fundamentalists and chartists. Obviously, changing $\phi$ does not change the pitchfork bifurcation boundary. However, for the Hopf bifurcation boundary $\omega=\omega^{*}$, we 
can show that there exists a $\phi^{*}$ such that $\omega^{*}$ is monotonically decreasing for $\phi<\phi^{*}$ and increasing for $\phi>\phi^{*}$. This feature is illustrated by the bifurcation boundaries in Figure 4.6(b) in the parameter space of $(\phi, \omega)$ for the chosen set of parameters. We see that for high $\omega$ (in fact $\omega$ near to 0.85 ), as $\phi$ increases from $\beta_{p}$, the fundamental steady state is stable initially, then becomes unstable through a Hopf bifurcation, and finally becomes stable again through a second Hopf bifurcation. This result is consistent with what we observed in the case of $\omega=1$ in Figure $4.4(\mathrm{~b})$.

In the second case when $\omega=0.5$ and $\beta_{f} \neq \beta_{c}$, a stability analysis can be found in Appendix 2, the result of which can be summarised as follows. When the portfolio managers weight the expectations of the fundamentalists and chartists equally, the stability of the fundamental equilibrium can be affected by the balance of the speeds of the mean-reversion of the fundamentalists and the extraploation of the chartists. When the portfolio managers react weakly to the expectations of the fundamentalists and chartists, the impact of the fundamentalists and chartists on the exchange rate is limited even if they are very active in forming their expectations. In contrast, if the portfolio managers dominate the market, the fundamental equilibrium becomes unstable and the active behaviour of the fundamentalists and chartists will accelerate the frequency of the market fluctuation due to the Hopf bifurcation. Therefore, the combination of the reaction of the portfolio managers to the heterogeneous expectations and the activities of the fundamentalists and chartists is an important factor in determining the evolution of the market exchange rate.

\section{A Stochastic Version of the Model}

The model analysed in the previous sections is entirely deterministic. However, in the real exchange rate market, there always exist some noise factors which have an important influence on the exchange rate dynamics. For example, fundamental noise (by which we mean noise in the underlying economic processes determining the fundamental value of the exchange rate) or market noise (this can best be thought of as noise impinging on the market due perhaps to the arrival of news events causing stochastic shifts in demand and supply-so called noise traders could be incorporated under this rubric), or both. For simplicity we focus on the case of market noise only. To capture this effect, the first equation in (2.12) is replaced by the stochastic differential equation

$$
d E=\left[H\left((1-\omega) X^{f}+\omega X^{c}\right)+\beta\left(R^{*}-R\right)\right] d t+\sigma_{E} d W_{E},
$$

where $W_{E}$ is a Wiener process on a probability space $(\Omega, \mathscr{F}, \mathscr{P})$ with zero drift and unit variance per unit time and $\sigma_{E}>0$ measures the intensity of the market noise.

In addition, in order to capture the different dynamics induced by the change in the weighting parameter in the deterministic model, the market fraction of the fundamentalists or chartists $(1-\omega$ or $\omega)$ will not be treated as constant but allowed to randomly 
change $^{8}$. Based on the analysis for the deterministic model in the previous sections, we see that changes in $\omega$ lead to the existence of three types of dynamics with a stable fundamental steady state (Case $\mathcal{F}$ ), two stable non-fundamental steady states (Case $\mathcal{P}$ ) and a stable limit cycle $($ Case $\mathcal{H})$, see Figure 4.5. To allow for all three types of behaviour, the market fraction $\omega$ is assumed to follow a simple discrete random choice process satisfying

$$
d \omega_{t}=\left(\xi-\omega_{t-}\right) d N_{t},
$$

where $N_{t}$ is a compound Poisson process with jump intensity $\lambda$. The process (5.2) can be rewritten as

$$
\omega_{t+}= \begin{cases}\xi & \text { with prob. } \lambda d t+o(d t), \\ \omega_{t-} & \text { with prob. } 1-\lambda d t+o(d t) .\end{cases}
$$

This means that when $d N_{t}=0$, the market fraction remains at the original level $\omega_{t-}$, while when $d N_{t}=1$, the market fraction $\omega_{t+}=\xi$ follows a Markov chain with three discrete states $\left\{\omega_{\mathcal{P}}, \omega_{\mathcal{F}}, \omega_{\mathcal{H}}\right\}$ and the transition probability matrix $\mathbf{P}$. The three states correspond to the three particular values of market dynamics in the cases $\mathcal{P}, \mathcal{F}$ and $\mathcal{H}$, respectively. Hence, the system $(2.12)$ can be modified to the stochastic differential system

$$
\left\{\begin{aligned}
d E & =\left[H\left(\left(1-\omega_{t}\right) X^{f}+\omega_{t} X^{c}\right)+\beta_{R}\left(R^{*}-R\right)\right] d t+\sigma_{E} d W_{E} \\
d P & =\rho\left[\beta_{0}-\beta_{1} Y-\beta_{2} R+\beta_{3}(E-P)\right] d t \\
d X^{f} & =\beta_{f}\left(R-R^{*}-X^{f}\right) d t \\
d X^{c} & =\beta_{c}\left\{\left[H\left(\left(1-\omega_{t}\right) X^{f}+\omega_{t} X^{c}\right)+\beta_{R}\left(R^{*}-R\right)-X^{c}\right] d t+\sigma_{E} d W_{E}\right\} \\
d \omega_{t} & =\left(\xi-\omega_{t-}\right) d N_{t}
\end{aligned}\right.
$$

where $R=\left(\alpha_{1} Y-M+P\right) / \alpha_{2}$. The system (5.3) implies that, when the exchange rate reacts to the noise factors, the chartists simultaneously adapt their belief to changes in the market. The fundamentalists rather focus on the fundamental information of the nominal interest rate differential, and they are not very sensitive to the immediate adjustments in the exchange rate.

\subsection{Empirical Evidence}

As mentioned in the introduction, a great deal of empirical research has uncovered many puzzles or anomalies in exchange rate markets, for example disconnection from fundamental factors, excess volatility, volatility clustering and fat tails. As a benchmark, we use daily Australian-US exchange rate market data coming from the Reserve Bank of Australia from Dec. 12, 1983 to Dec. 31, 2009. The exchange rate is measured by

\footnotetext{
${ }^{8}$ Another mechanism for changing weights could be based on some fitness measure, which has been widely used in asset pricing models with heterogeneous beliefs following Brock and Hommes (1998) and De Grauwe and Grimaldi (2006) in exchange rate markets.
} 
the amount of AUD for 1 unit of USD. In total, there are 6,577 daily exchange rate observations. Figure 5.1(a) shows the time series of the real log-exchange rate (LER). We observe that the AUD fluctuates a great deal over the years 1985-1986, 2000-2001 and 2006-2009 (the first 800, the 3,500-4,500 and the 5,500-6,577 data points) and is relatively stable over the rest of time period. The corresponding distribution of the logexchange rate in Figure 5.1(c) displays two peaks, one high peak near 0.25 and low one near 0.65. The returns of the exchange rate in Figure 5.1(b) show the switching between high volatility and low volatility that is typical of many financial return time series. Also the density distribution of the returns in Figures 5.1(d) and (e) is not normal, having very fat tails. The absolute and squared returns in Figures 5.1(f)-(h) display rapidly decreasing positive ACs but the return itself has insignificant ACs.

Figure 5.1 here

\subsection{Stochastic Simulations of the Model and Statistical Anal- ysis}

For our stochastic model (5.3), we regard $\omega$ as the bifurcation parameter. Let $\mu=$ $1, \beta_{R}=0.85, \beta_{f}=0.8$ and $\beta_{c}=0.8$ as in Figure 4.5. Then we know from subsection 4.3 that $\omega_{\mathcal{P}}^{*}=0.15$ and $\omega_{\mathcal{H}}^{*} \approx 0.8286$ are the pitchfork and Hopf bifurcation points of the fundamental steady state, respectively. That is, when $\omega<\omega_{\mathcal{P}}^{*}$ (for example $\omega=0.10$ ), the fundamental steady state is unstable and two non-fundamental steady states appear $\left(\right.$ Case $\mathcal{P}$ ). When $\omega>\omega_{\mathcal{H}}^{*}$ (for example $\omega=0.85$ ), the fundamental steady state is unstable and a stable limit cycle exists (Case $\mathcal{H}$ ). When $\omega_{\mathcal{P}}^{*}<\omega<\omega_{\mathcal{H}}^{*}$ (for example $\omega=0.25)$, the fundamental steady state is stable (Case $\mathcal{F})$.

To examine the effect of random disturbance on the deterministic dynamics, we assume that the random market fraction $\omega$ satisfies (5.2) in which $\xi$ follows a Markov chain with three states $\{0.10,0.25,0.85\}$. These three sates correspond the three typical dynamical regions that the analysis of Section 4 has shown that the model displays, namely Cases $\mathcal{P}, \mathcal{F}, \mathcal{H}$ respectively. The transition probability matrix among the different states is assumed to be given by

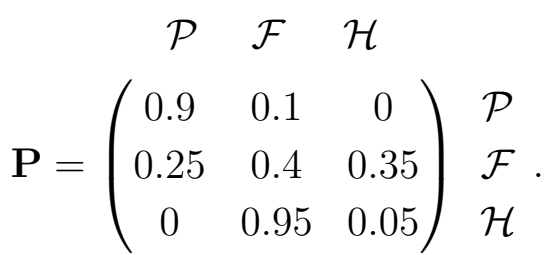

The principle behind this choice is that the weights are not allowed to have extreme jumps, say, from $\omega=0.10$ to $\omega=0.85$ without going through $\omega=0.25$. The size of the noises is given by $\sigma_{E}=0.0158$ per trading period and $\lambda=0.05$ (meaning that, on average, there is one jump for every 20 trading periods). Figure 5.2 displays the time series and statistical characteristics based on the system (5.3). 
NOTE. As required by the referee report, we need to have a figure, say Fig. ??, of time series of both the log exchange rate and $\omega$, together with the bifurcation values of $\omega, \omega_{P}^{*}=0.15$ and $\omega_{H}^{*}=0.8286$, to illustrate the impact of the underlying deterministic dynamics on the stochastic nature of the exchange rate.

Figure ?? here

Figure 5.2 here

Figure 5.2(a) shows a typical time series of the log-exchange rate and Figure 5.2(b) shows the corresponding return series. They display persistent deviation of the market exchange rate from the (constant) fundamental exchange rate and irregular switching between phases of low volatility with returns close to zero and phases of high volatility. This is because the weight between the fundamentalists and chartists changes stochastically. For different weights, the exchange rate has different stability properties. When $\omega=0.25$, the fundamental exchange rate converges to the fundamental steady state equilibrium and the corresponding time series shows such a converging tendency. However, when $\omega=0.10$, the fundamental equilibrium is unstable and another two nonfundamental steady states co-exist. In this case, the time series undergoes a deviation from the fundamental equilibrium. When $\omega=0.85$, there is a stable limit cycle around the unstable fundamental equilibrium for the deterministic model and the time series shows a fluctuation around the fundamental equilibrium level. Not surprisingly, adding the dynamical noise to the system destroys the regularity of the exchange rate around the (non-)fundamental steady state, or the cycle and leads to an irregular switching between phases of low volatility (where the fundamental equilibrium is stable) and phases of high volatility (where the fundamental equilibrium is unstable), and of deviation from the underlying fundamental equilibrium most of the time. Furthermore, the density of the log-exchange rate is bimodal as observed in Figure 5.2(c) and the return of the exchange rate in Figure 5.2(b) shows volatility clustering. In addition, the absolute and squared returns in Figures 5.2(g) and (h) display strong positive autocorrelations (ACs), but the ACs of the return in Figure 5.2(f) are insignificant (except the first few time lags). The distribution of the exchange rate return in Figures 5.2(d) and (e) is non-normal with fat tails. These effects correspond to most of the stylised facts observed in exchange rate markets.

Comparing the characteristics in Figures 5.2 and 5.1, we can see that our simple heterogeneous expectations model can replicate the broad features of realistic time series and some stylised facts in exchange rate markets, for example, deviation from fundamental factors, bimodal density of log-exchange rate, volatility clustering and fat tails for returns. The autocorrelation patterns also show the broad tendencies of those observed in the actual times series but clearly these are not as satisfactory. Overall our results indicate that heterogeneous beliefs in exchange rate markets can potentially explain the 
puzzles and anomalies of exchange rates to a certain extent and the interaction between the fundamentalists and chartists is a potential source of the mechanism generating some of those characteristics.

\section{Conclusion}

Motivated by the challenge faced by traditional macroeconomic exchange rate models in explaining an increasing number of anomalies and puzzles uncovered in empirical research, and empirical evidence that many popular technical trading strategies are used in exchange markets, this paper extends the classical Dornbousch exchange rate model with homogeneous expectation to incorporate heterogeneous beliefs and bounded rationality into a dynamical model of exchange rates in a continuous time framework.

Essentially, the heterogeneity is characterised by the expectations of the portfolio managers, which are a weighted average of the expectations of the fundamentalists and chartists, and the bounded rationality is characterised by the different information of the heterogeneous investors and the $S$-shaped expectation function of the portfolio managers. The model not only inherits the classical saddle-point instability of the Dornbousch model and the stabilising effect of the sluggish adjustment in exchange rates of the Gray and Turnovsky model, but also introduces many new desirable features. These include the stability of the fundamental equilibrium, the existence and stability of nonfundamental equilibria, and different exchange rate dynamics induced by different types of bifurcations.

The analysis of the model highlights the different roles played by the macro fundamental factor and the combination of the heterogeneous expectations. In general, the macro fundamental factor is a stabilising force in the market, whilst the speculative activities from the fundamentalists and chartists can destabilise the fundamental equilibrium of the exchange rate. In particular, the macro fundamental factor can stabilise an otherwise unstable market when the macro fundamental factor dominates speculative expectations. When the market fundamental equilibrium is unstable, the fundamentalist expectation tends to cause the exchange rate to deviate from the fundamental towards a non-fundamental equilibrium level characterised by a pitchfork bifurcation, whilst the chartist expectation causes the exchange rate to fluctuate periodically around the fundamental equilibrium characterised by a Hopf bifurcation. This difference is clearly demonstrated in two special cases where the expectations of the portfolio managers follow the view of either the fundamentalists or the chartists. In the general case, when the expectations of the portfolio managers are a weighted average of the heterogeneous expectations, the exchange rate displays a mixture of these two different features dominated by either one of the expectation schemes. Also, a mixture of the two schemes can stabilise an otherwise unstable fundamental steady state and this stabilising effect of combining different types of expectations is also shared by asset pricing models with 
heterogeneous beliefs. In addition, it is shown that the balance between the macro fundamental factor and the expectations of the portfolio managers is an important factor in determining the exchange rate market evolution.

For the corresponding stochastic model, noise processes are introduced through the market noise in terms of the change of the log-exchange rate (equation 5.1) and the random switching of the weights of the two heterogeneous expectations (equation 5.2). Numerical simulations show that our simple heterogeneous expectations model can replicate typical time series and some stylised facts observed in exchange rate markets, including deviation from fundamental factors, bimodal density, volatility clustering and fat tails.

Here in order to focus just on the underlying mechanism driving the exchange rate, namely the different stability regimes, we have simply relied on stochastic switching between the various strategies. The next step is to adopt some of the more sophisticated switching mechanisms introduced in the literature such as that of Brock and Hommes (1997, 1998) involving switching populations or that of Lux (1997) involving transition probabilities. Incorporation of such switching mechanisms should lead to better realism in the generated time series, however this task we leave to further research.

\section{Acknowledgement}

An earlier version of this paper was presented at the 2008 SNDE (San Francisco) and CEF 2008 (Paris) conferences and seminars at UTS and Deakin University. Financial support from the Australian Research Council (ARC) under a Discovery Grant (DP0773776) and a Faculty Research Grant at UTS is greatly acknowledged. We would like to thank the editor of this special issue, Didier Sornette, and a referee for their helpful comments and valuable suggestions. The usual caveat applies.

\section{Appendix 1. Proofs}

Proof of Theorem 4.1: At the fundamental steady state, the corresponding Jacobian matrix is given by

$$
A=\left(\begin{array}{cccc}
0 & -\frac{\beta_{R}}{\alpha_{2}} & \mu(1-\omega) & \mu \omega \\
\rho \beta_{3} & -\rho\left(\beta_{3}+\frac{\beta_{2}}{\alpha_{2}}\right) & 0 & 0 \\
0 & \frac{\beta_{f}}{\alpha_{2}} & -\beta_{f} & 0 \\
0 & -\frac{\beta_{R} \beta_{c}}{\alpha_{2}} & \beta_{c} \mu(1-\omega) & \beta_{c}(\mu \omega-1)
\end{array}\right)
$$

the characteristic equation of which has the form

$$
p(x):=x^{4}+d_{1} x^{3}+d_{2} x^{2}+d_{3} x+d_{4}=0,
$$

where $d_{i}(i=1, \ldots, 4)$ are defined in (4.2). Applying the Routh-Hurwitz Theorem (see Zadeh and Desoer, 1963), we obtain the result on the stability of the fundamental steady state. 
By the Routh-Hurwitz criterion, Theorems 4.2, 4.3 and 4.4 can be proven. Here we just give the proof of Theorem 4.2 as an example.

Proof of Theorem 4.2: For the system (4.4), the characteristic equation of the fundamental steady state $\mathcal{S}_{0}$ becomes

$$
p(x)=x^{3}+a_{01} x^{2}+a_{02} x+a_{03},
$$

where

$$
a_{01}:=\beta_{f}+\beta_{p}, \quad a_{02}:=\beta_{p} \beta_{f}+\beta_{e} \beta_{R}, \quad a_{03}:=\beta_{e} \beta_{f}\left(\beta_{R}-\mu\right) .
$$

Let $\Delta_{03}:=a_{01} a_{02}-a_{03}=\beta_{p} \beta_{f}^{2}+\beta_{p}^{2} \beta_{f}+\beta_{p} \beta_{e} \beta_{R}+\beta_{e} \mu \beta_{f}$, then $\mathcal{S}_{0}$ is LAS if and only if 9

$$
a_{01}>0, \quad a_{03}>0, \quad \Delta_{03}>0 .
$$

It is obvious that $a_{01}$ and $\Delta_{03}$ are always positive and the fundamental steady state loses its stability only at $a_{03}=0$, that is $\beta_{R}=\mu$. At $\beta_{R}<\mu$, two non-fundamental steady states $\mathcal{S}_{ \pm}=\left(E^{*}+\left(\frac{\beta_{2}}{\beta_{3}}+\alpha_{2}\right) X_{ \pm}^{*}, P^{*}+\alpha_{2} X_{ \pm}^{*}, X_{ \pm}^{*}\right)$ appear, where $X_{ \pm}^{*}$ satisfy $H\left(X_{ \pm}^{*}\right)=$ $\beta_{R} X_{ \pm}^{*}, X_{ \pm}^{*} \neq 0$ and $H^{\prime}\left(X_{ \pm}^{*}\right)<\beta_{R}$, that is to say a pitchfork bifurcation occurs. At the non-fundamental steady states $\mathcal{S}_{ \pm}$, their stability properties are determined by the eigenvalues that are the solutions of

$$
\tilde{p}(y)=y^{3}+\tilde{a}_{01} y^{2}+\tilde{a}_{02} y+\tilde{a}_{03}
$$

where

$$
\tilde{a}_{01}:=\beta_{f}+\beta_{p}, \quad \tilde{a}_{02}:=\beta_{p} \beta_{f}+\beta_{e} \beta_{R}, \quad \tilde{a}_{03}:=\beta_{e} \beta_{f}\left(\beta_{R}-H^{\prime}\left(X_{ \pm}^{*}\right)\right),
$$

and $\tilde{\Delta}_{03}:=\tilde{a}_{01} \tilde{a}_{02}-\tilde{a}_{03}$. Since $\tilde{a}_{0 i}(i=1,2,3)$ and $\tilde{\Delta}_{03}$ are all positive, $\tilde{p}(y)=0$ only has negative eigenvalues, that is $\mathcal{S}_{ \pm}$is LAS.

\section{Appendix 2. The stability analysis of Case 2: $\omega=0.5$ and $\beta_{f} \neq \beta_{c}$ in subsection 4.3}

In this case, the stability of the fundamental equilibrium is determined by the balance of the expectations of the fundamentalists and chartists. This is characterised by the sign change of the discriminants $d_{i}(i=1,2,4)$ given in (4.2), and $\Delta_{4}=c_{0} \beta_{c}^{3}+c_{1} \beta_{c}^{2}+c_{2} \beta_{c}+c_{3}$, where

$$
\begin{aligned}
& c_{0}=\frac{(2-\mu)^{2}}{8}\left[(2-\mu) \beta_{p} \beta_{f}^{2}+\left((2-\mu) \beta_{p}^{2}+\beta_{e} \mu\right) \beta_{f}+2 \beta_{p} \beta_{e} \beta_{R}\right], \\
& c_{3}=\frac{\left(2 \beta_{p} \beta_{f}^{2}+\left(\beta_{e} \mu+2 \beta_{p}^{2}\right) \beta_{f}+2 \beta_{p} \beta_{e} \beta_{R}\right)\left(2 \beta_{R}-\mu\right) \beta_{f} \beta_{e}}{4},
\end{aligned}
$$

\footnotetext{
${ }^{9}$ Here the conditions (A.3) are equivalent to (4.1) under $\omega=0$ and especially, $a_{03}=0$ corresponds to $d_{4}=0$ and $\Delta_{03}=0$ corresponds to $\Delta_{4}=0$.
} 
and $c_{1}, c_{2}$ are also some polynomials and at $\mu=2, c_{1}=-\beta_{e}^{2} \beta_{R}^{2}<0$. As before, $d_{4}=0$ and $\Delta_{4}=0$ correspond to the pitchfork and Hopf bifurcation boundaries, respectively. Note that the changes in both $\beta_{f}$ and $\beta_{c}$ do not affect the sign of $d_{4}=\beta_{e} \beta_{c} \beta_{f}\left(\beta_{R}-\mu / 2\right)$. Hence we assume $d_{4}>0$, that is, $\mu<2 \beta_{R}$ and hence $c_{3}>0$. Thus, we only need to focus on the sign change of $\Delta_{4}$. At $\beta_{c}=0, \Delta_{4}>0$ but $\Delta_{4}$ changes as $\beta_{f}$ and $\beta_{c}$ increase.

(i) For $\mu<2$ and $\beta_{f}>0$, we have $c_{0}>0$. Note that $\lim _{\beta_{c} \rightarrow+\infty} \Delta_{4}=+\infty$, that is to say that there exists $\beta_{c}^{*} \geq 0$ which depends on $\beta_{f}$ such that $\Delta_{4}>0$ for $\beta_{c}>\beta_{c}^{*}$. Correspondingly the fundamental steady state equilibrium $\mathcal{S}_{0}$ is stable for $\beta_{c}>\beta_{c}^{*}$ and $\mu<\min \left\{2,2 \beta_{R}\right\}$.

(ii) For $\mu=2, c_{0}=0$ and $c_{1}<0$. So for any $\beta_{f}>0$, there exists $\beta_{c}^{*}=\beta_{c}^{*}\left(\beta_{f}\right)>0$ such that $\Delta_{4}<0$ for $\beta_{c}>\beta_{c}^{*}$. Correspondingly the fundamental steady state equilibrium $\mathcal{S}_{0}$ is unstable for $\beta_{c}>\beta_{c}^{*}$ and $\mu=2$.

(iii) For $\mu>2$, there exist $\beta_{f}^{*}>0$ such that when $\beta_{f}>\beta_{f}^{*}, c_{0}<0$. At the same time, $\lim _{\beta_{c} \rightarrow+\infty} \Delta_{4}=-\infty$, that is to say that there exists $\beta_{c}^{*}=\beta_{c}^{*}\left(\beta_{f}\right)>0$ such that since $\beta_{c}>\beta_{c}^{*}$, it follows that $\Delta_{4}<0$. Correspondingly the fundamental steady state equilibrium $\mathcal{S}_{0}$ is unstable for $\beta_{f}>\beta_{f}^{*}$ and $\beta_{c}>\beta_{c}^{*}$ and $2<\mu<2 \beta_{R}$.

Essentially, the local stability of the fundamental equilibrium is determined by the sign of $\Delta_{4}$. When the intensity of the adjustment of the portfolio managers on the weighted depreciation rate of the fundamentalists and chartists is comparatively weak (so that $\mu<\min \left\{2,2 \beta_{R}\right\}$ ), a strong extrapolation from the chartists (so that $\beta_{c}>\beta_{c}^{*}=$ $\beta_{c}^{*}\left(\beta_{f}\right)$ for any given speed of the mean-reversion of the fundamentalists) can locally stabilise the fundamental equilibrium. In contrast, if the portfolio managers dominate the market (so that $\mu \geq 2$ ), the fundamental equilibrium becomes unstable and the active behaviour of the fundamentalists and chartists will accelerate the frequency of the market fluctuation due to the Hopf bifurcation. 


\section{References}

Allen, H. and Taylor, M. P. (1990), 'Charts, noise and fundamentals in the London foreign exchange market', The Economic Journal 100, 49-59.

Baxter, M. and Stockman, A. (1989), 'Business cycles and the exchange rate regime: some international evidence', Journal of Monetary Economics 23, 377-400.

Brock, W. and Hommes, C. (1997), 'A rational route to randomness', Econometrica 65, 1059-1095.

Brock, W. and Hommes, C. (1998), 'Heterogeneous beliefs and routes to chaos in a simple asset pricing model', Journal of Economic Dynamics and Control 22, 1235-1274.

Chiarella, C., Dieci, R. and Gardini, L. (2002), 'Speculative behaviour and complex asset price dynamics: a global analysis', Journal of Economic Behavior and Organization 49, 173-197.

Chiarella, C., Dieci, R. and He, X. (2009), Heterogeneity, Market Mechanisms and Asset Price Dynamics, Elsevier, pp. 277-344. in Handbook of Financial Markets: Dynamics and Evolution, Eds. Hens, T. and K.R. Schenk-Hoppe.

Chiarella, C. and He, X. (2002), 'Heterogeneous beliefs, risk and learning in a simple asset pricing model', Computational Economics 19, 95- 132.

Chinn, M. D. and Frankel, J. A. (2002), Survey data on exchange rate expectations: More currencies, more horizons, more tests, in D. G. Dickinson and W. A. Allen, eds, 'Monetary Policy, Capital Flows and Exchange Rates: Essays in honour of Maxwell Fry', Routledge, London and New York, chapter 9, pp. 145-167.

De Grauwe, P. and Grimaldi, M. (2005a), Heterogeneity of agents and the exchange rate: a nonlinear approach, in P. De Grauwe, ed., 'Exchange Rate Economics: Where Do We Stand?', MIT Press, Cambridge, pp. 125-167.

De Grauwe, P. and Grimaldi, M. (2005b), 'Heterogeneity of agents, transactions costs and the exchange rate', Journal of Economic Dynamics and Control 29, 691-719.

De Grauwe, P. and Grimaldi, M. (2006), 'Exchange rate puzzles. a tale of switching attractors', European Economic Review 50, 1-33.

Dornbusch, R. (1976), 'Expectations and exchange rate dynamics', Journal of Political Economy 84(6), 1161-1176.

Flood, R. and Rose, A. (1995), 'Fixing exchange rates: a virtual quest for fundamentals', Journal of Monetary Economics 36, 3-37. 
Frankel, J. A. and Froot, K. A. (1986), 'Understanding the US dollar in the eighties: The expectations of chartists and fundamentalists', The Economic Record, pp. 24-38. Special issue.

Frankel, J. A. and Froot, K. A. (1990a), Chartists, fundamentalists and the demand for dollars, in A. S. Courakis and M. P. Taylor, eds, 'Private behaviour and government policy in interdependent economies', Oxford University Press, New York, pp. 73126.

Frankel, J. A. and Froot, K. A. (1990b), 'The rationality of the foreign exchange rate. chartists, fundamentalists and trading in the foreign exchange market', American Economic Review 80(2), 181-185. AEA Papers and Proceedings.

Gray, M. R. and Turnovsky, S. J. (1979), 'The stability of exchange rate dynamics under perfect myopic foresight', International Economic Review 20(3), 643-660.

Hommes, C. (2006), Heterogeneous Agent Models in Economics and Finance, Vol. 2 of Handbook of Computational Economics, North-Holland, pp. 1109-1186. in Agentbased Computational Economics, Eds. Tesfatsion, L. and K.L. Judd.

Hommes, C. and Wagener, F. (2009), Complex Evolutionary Systems in Behavioral Finance, number Chapter 4 in 'Handbooks in Finance', North-Holland, pp. 217-276. in Handboof of Financial Markets: Dynamics and Evolution, Eds. Hens, T. and K. Schenk-Hoppe.

Ito, T. (1990), 'Foreign exchange rate expectations: Micro survey data', American Economic Review 80, 434-449.

Kuznetsov, Y. A. (2004), Elements of Applied Bifurcation Theory, Third edn, SpringerVerlag, New York.

LeBaron, B. (2006), Agent-based Computational Finance, Vol. 2 of Handbook of Computational Economics, North-Holland, pp. 1187-1233. in Agent-based Computational Economics, Eds. Tesfatsion, L. and K.L. Judd.

Lux, T. (1997), 'Time variation of second moments from a noise trader/infection model', Journal of Economic Dynamics and Control 22, 1-38.

Lux, T. (1998), 'The socio-economic dynamics of speculative markets: Interacting agents, chaos, and fat tails of return distributions', Journal of Economic Behavior and Organization 33, 142-165.

Lux, T. (2009), Stochastic Behavioural Asset Pricing and Stylized Facts, Elsevier, pp. 161-215. in Handbook of Financial Markets: Dynamics and Evolution, Eds. Hens, T. and K.R. Schenk-Hoppe. 
Lux, T. and Marchesi, M. (2001), 'Volatility clustering in financial markets: A microsimulation of interacting agents', Journal of Theoretical and Applied Finance 3, 675702.

Lyons, R. (2001), The Microstructure Approach to Exchange Rates, MIT Press, Cambridge.

Meese, R. and Rogoff, K. (1983), 'Empirical exchange rate models of the seventies', Journal of International Economics 14, 3-24.

Menkhoff, L. (1998), 'The noise trading approach-questionnaire evidence from foreign exchange', Journal of International Money and Finance 17, 547-564.

Menkhoff, L. and Taylor, M. (2007), 'The obstinate passion of foreign exchange professionals: technical analysis', Journal of Economic Literature 45, 936-972.

Reitz, S. and Taylor, M. (2008), 'The coordination channel of foreign exchange intervention: a nonlinear microstructure analysis', European Economic Review 52, 55-76.

Taylor, M. P. and Allen, H. (1992), 'The use of technical analysis in the foreign exchange market', Journal of International Money and Finance 11, 304-314.

Westerhoff, F. (2003), 'Expectations driven distortions in the foreign exchange market', Journal of Economic Behavior and Organization 51, 389-412.

Westerhoff, F. (2010), Handbook of Research on Complexity, Edward Elgar, Cheltenham, chapter Exchange rate dynamics: A nonlinear survey, in press.

Westerhoff, F. and Reitz, S. (2003), 'Nonlinearities and cyclical behavior: The role of chartists and fundamentalists', Studies in Nonlinear Dynamics and Econometrics $7(4)$, article no. 3 .

Williamson, J. (1985), The exchange rate system, Vol. 5, Washington, D.C.: Institute for International Economics.

Zadeh, L. A. and Desoer, C. A. (1963), Linear System Theory: The State Space Approach, McGraw-Hill Series in System Science, McGraw-Hill, USA. 


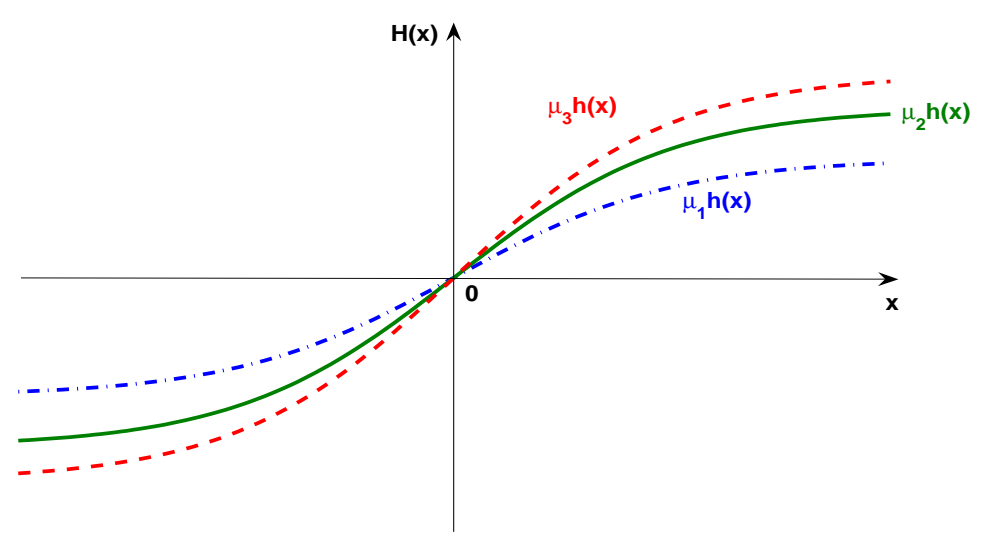

Figure 2.1: The expected exchange depreciation rate function $H$ of the market expectation with different parameter $\mu_{i}(i=1,2,3)$ where $0<\mu_{1}<\mu_{2}<\mu_{3}$. 


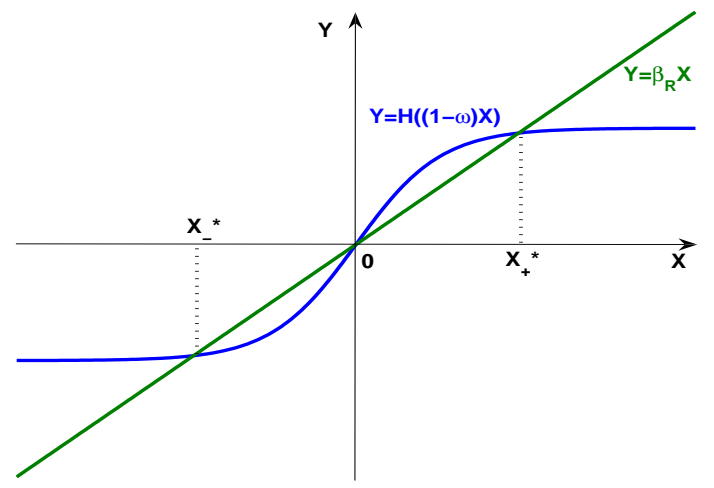

Figure 3.1: The three solutions of $H((1-\omega) X)=\beta_{R} X$ for $\mu(1-\omega)>\beta_{R}$. 


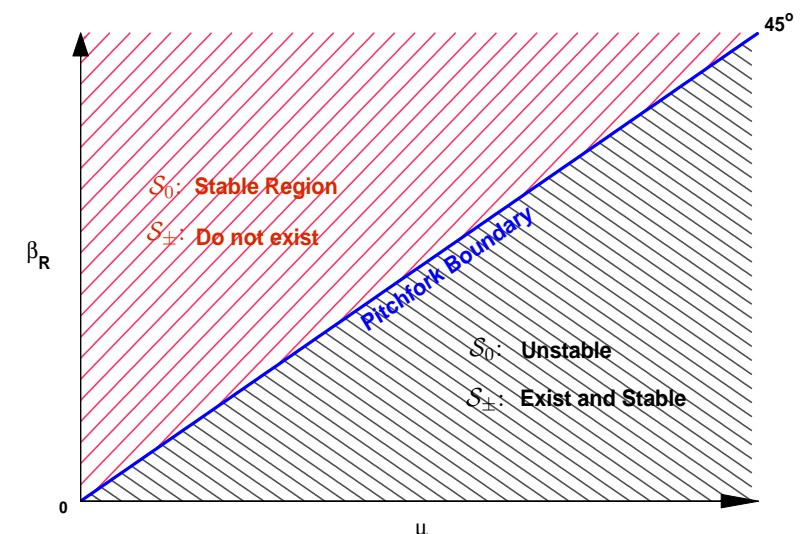

Figure 4.1: Parameter regions of the stable and unstable fundamental steady states in $\left(\mu, \beta_{R}\right)$ space and the corresponding pitchfork bifurcation boundary. 


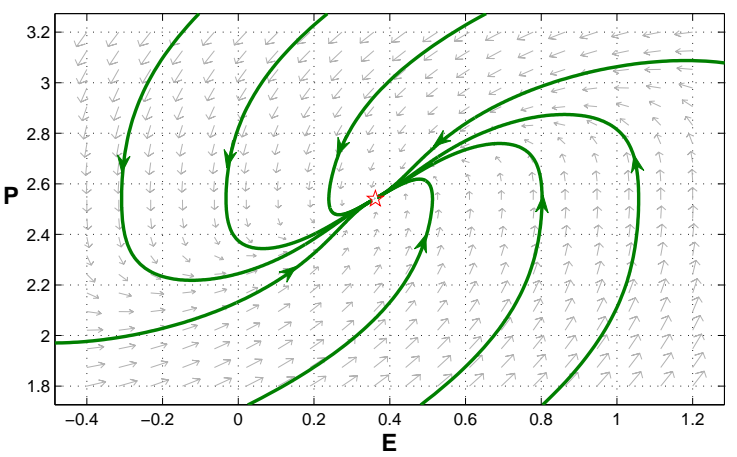

(a) $\beta_{R}=1.2$

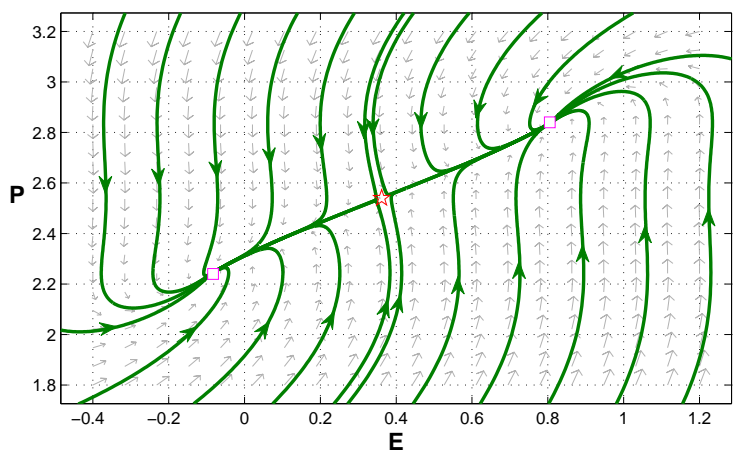

(b) $\beta_{R}=0.85$

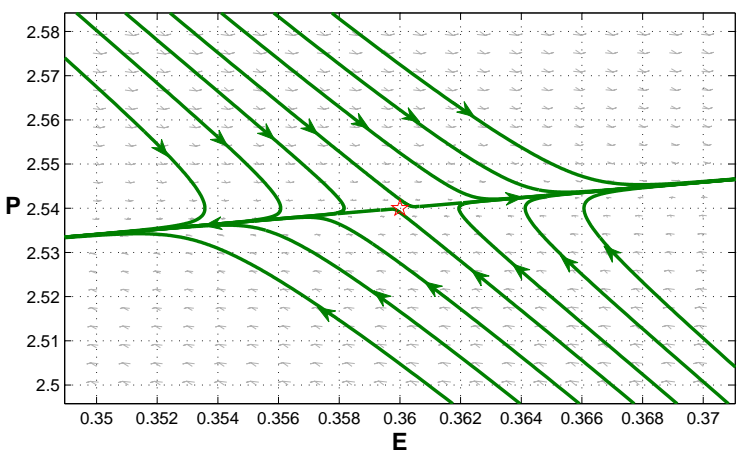

(c) $\beta_{R}=0.85$

Figure 4.2: The phase plots of $(E, P)$ and the stability analysis when $\beta_{f}=\infty$ and $\mu=1$. (a) When $\beta_{R}>\mu$, there is a unique and stable fundamental equilibrium $\left(E^{*}, P^{*}\right)$; (b) when $\beta_{R}<\mu$, the fundamental equilibrium $\left(E^{*}, P^{*}\right)$ becomes unstable and two locally stable non-fundamental steady states appear; (c) looking at the picture in (b) very locally and seeing just the saddle-point nature of the unstable equilibrium. Here the star ( $)$ denotes $\left(E^{*}, P^{*}\right)$ and the squares $(\square)$ denote the non-fundamental equilibria. 


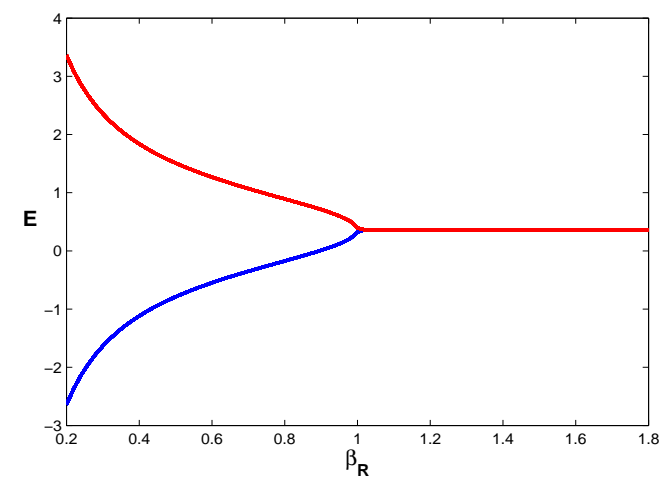

(a) Bifurcation plot in parameter $\beta_{R}$.

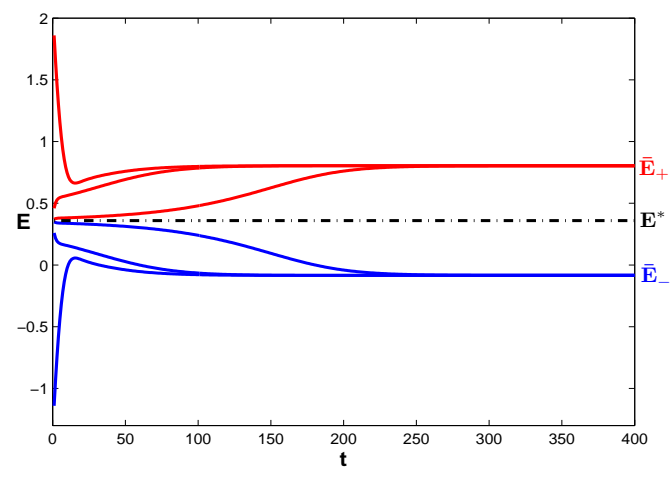

(b) Convergence of the exchange rates

Figure 4.3: When $0.8=\beta_{f}<\infty$, we take $\mu=1$ as in Figure 4.2 and show (a) the bifurcation plot of the log-exchange rate with respect to the parameter $\beta_{R}$ and pitchfork bifurcation; (b) the evolution of the log-exchange rate $E(t)$ from different initial values when $\beta_{R}=0.85\left(<\beta_{R}^{*}=\mu\right)$. 

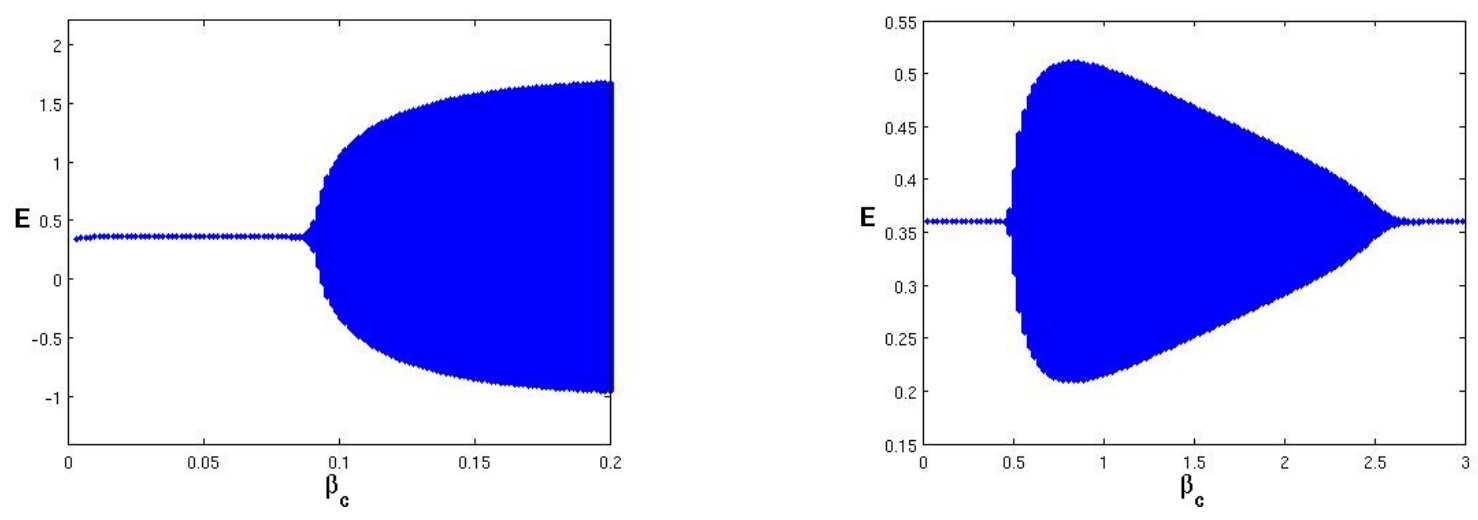

(a)

Figure 4.4: For $\omega=1$, the bifurcation plots of the log-exchange rate with respect to the parameter $\beta_{c}$ for $\beta_{R}=10$ and (a) $\mu=2>1$; (b) $0.5=\mu<1$. 


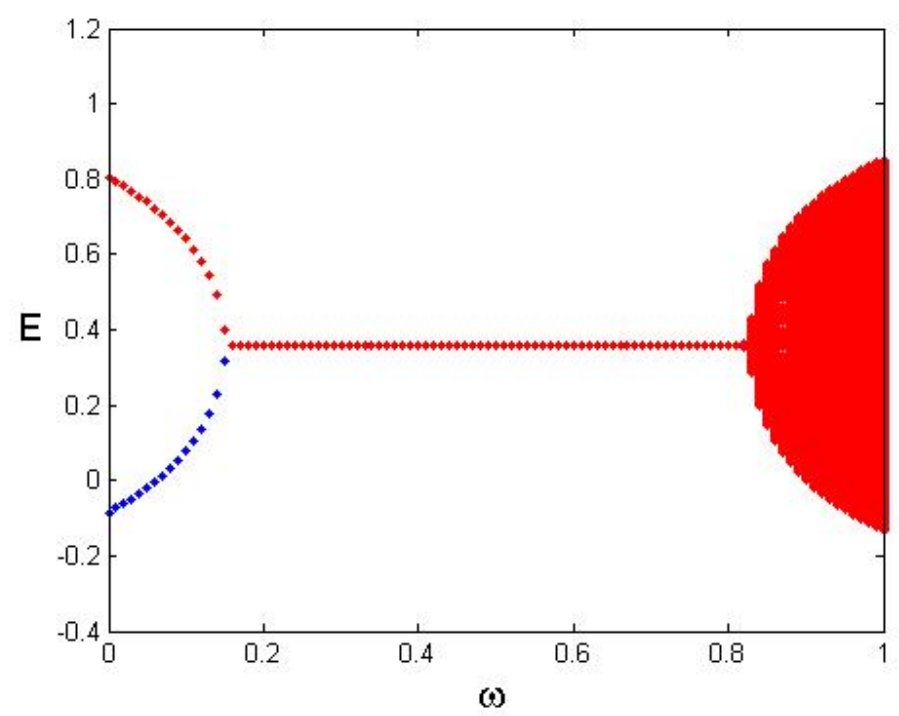

Figure 4.5: The bifurcation of the log-exchange rate $E$ in terms of the parameter $\omega \in$ $(0,1)$ for $\mu=1, \beta_{R}=0.85, \beta_{f}=0.8$ and $\beta_{c}=0.8$. The fundamental steady state undergoes a pitchfork bifurcation at $\omega=1-\beta_{R}=0.15$, and a Hopf bifurcation at $\omega=\omega^{*}=0.8286$. 


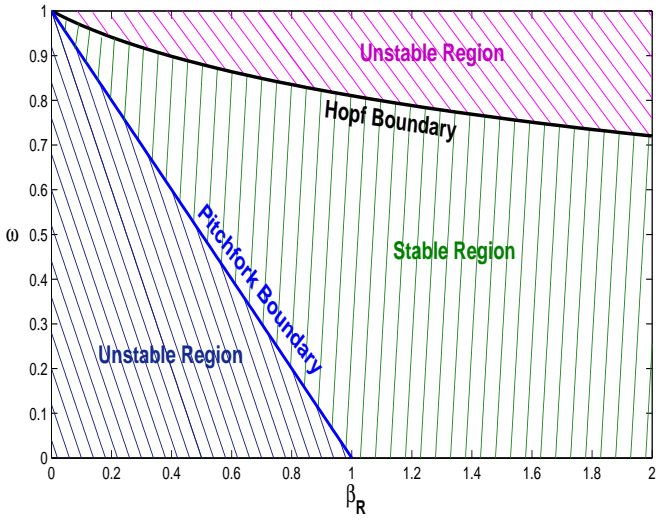

(a) $\phi=0.8$

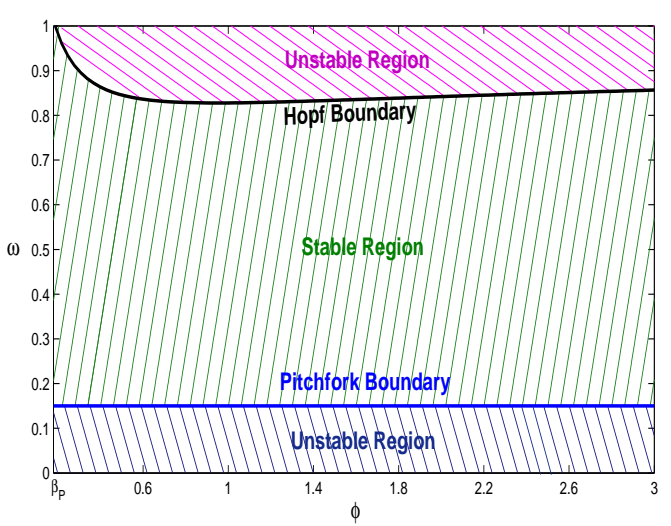

(b) $\beta_{R}=0.85$

Figure 4.6: Stability regions and bifurcation boundaries; (a) in the $\left(\beta_{R}, \omega\right)$ plane, and (b) in the $(\phi, \omega)$ plane. Here $\beta_{f}=\beta_{c}=\phi, \mu=1$ and $\beta_{p}<\phi$. 


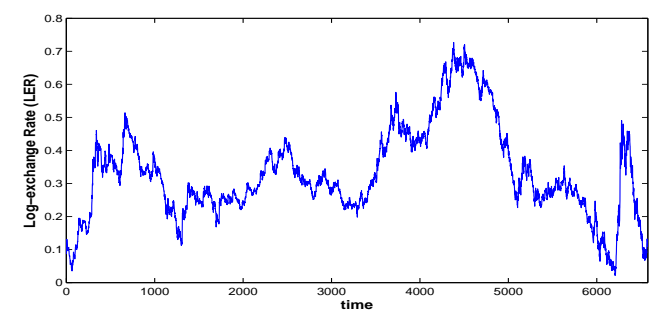

(a) Time series of log-exchange rate (LER)

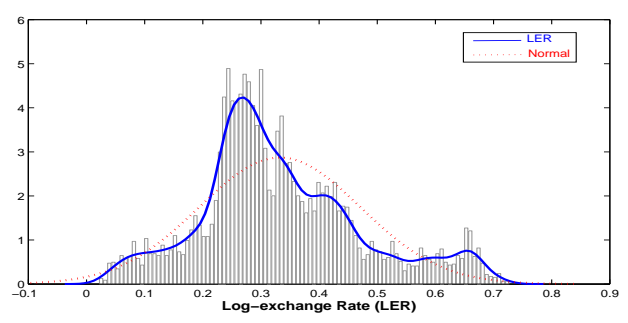

(c) Density of LER

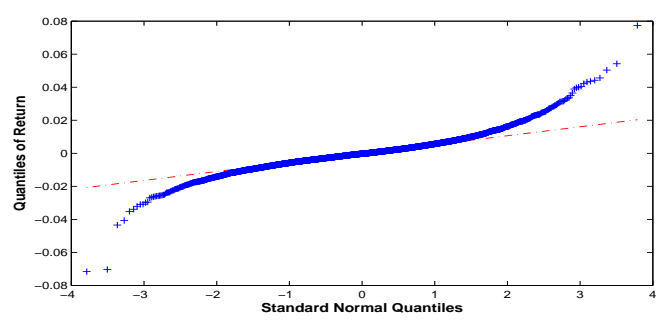

(e) QQ-plot of $r$

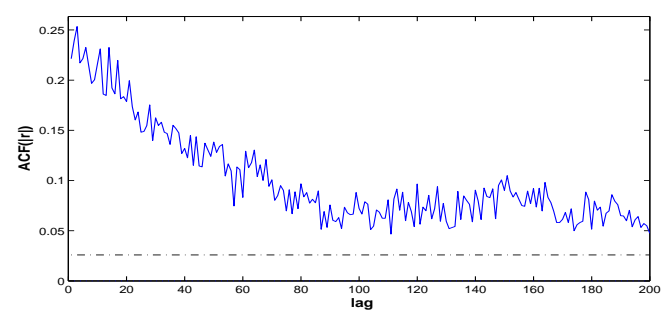

(g) ACF of $|r|$

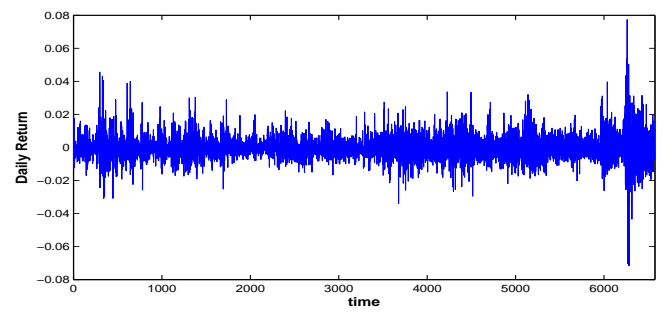

(b) Time series of daily return $(r)$

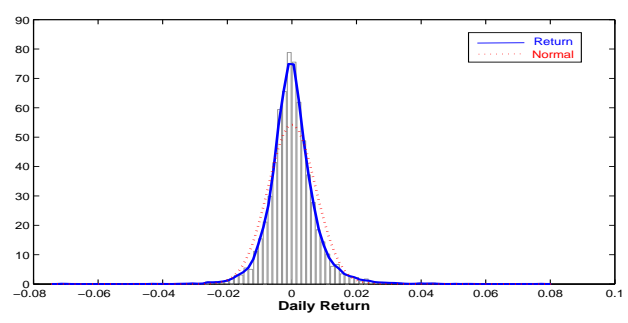

(d) Density of $r$

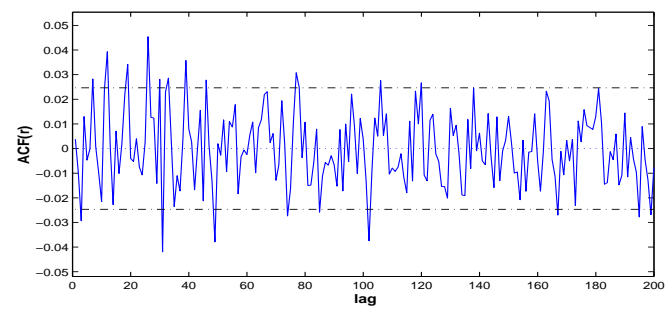

(f) ACF of $r$

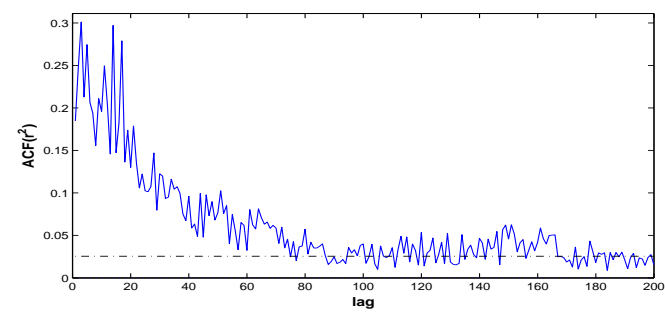

(h) ACF of $r^{2}$

Figure 5.1: Statistical analysis of the exchange rate daily data between the Australia dollar and the US dollar from Dec. 12, 1983 to Dec. 31, 2009. 


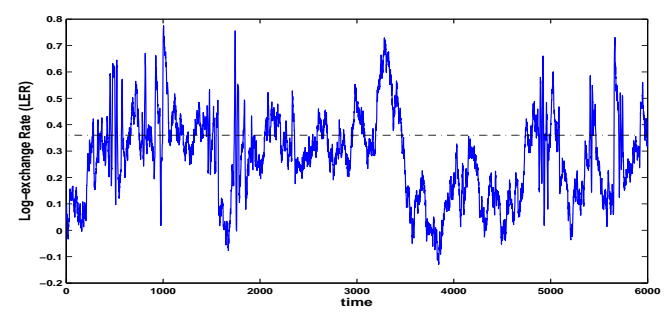

(a) Time series of log-exchange rate (LER)

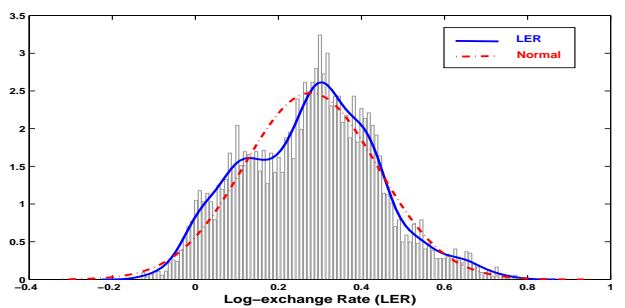

(c) Density of LER

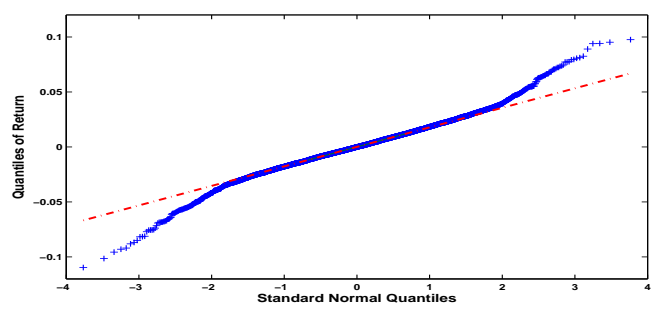

(e) QQ-plot of $r$

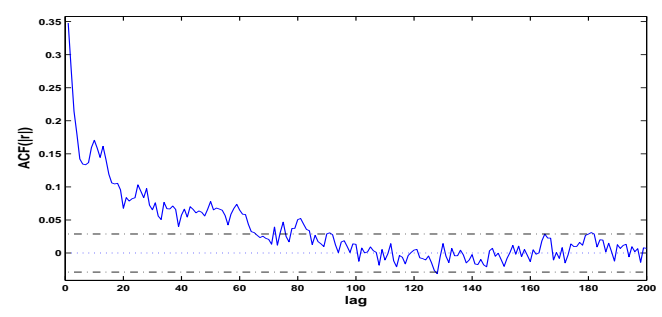

(g) ACF of $|r|$

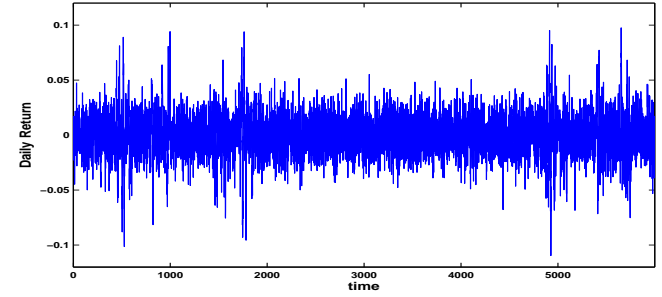

(b) Time series of daily return $(r)$

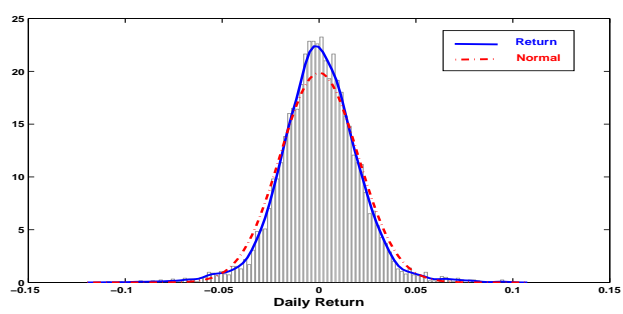

(d) Density of $r$

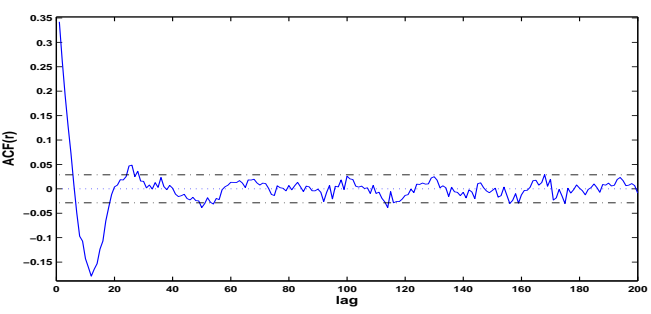

(f) $\mathrm{ACF}$ of $r$

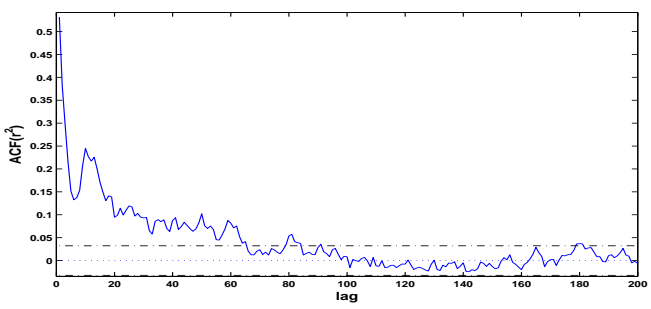

(h) $\mathrm{ACF}$ of $r^{2}$

Figure 5.2: Statistical analysis of the simulation results. 